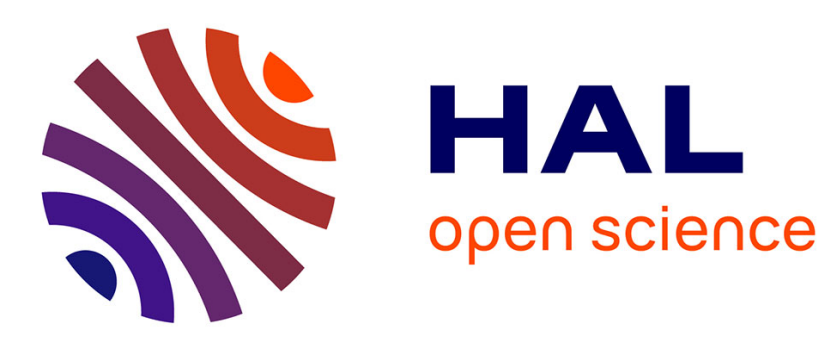

\title{
Self-Regulating Processes
}

Olivier Barrière, Antoine Echelard, Jacques Lévy Véhel

\section{To cite this version:}

Olivier Barrière, Antoine Echelard, Jacques Lévy Véhel. Self-Regulating Processes. Electronic Journal of Probability, 2012, 10.1214/EJP.v17-2010 . hal-00749742

\section{HAL Id: hal-00749742 \\ https://hal.inria.fr/hal-00749742}

Submitted on 8 Nov 2012

HAL is a multi-disciplinary open access archive for the deposit and dissemination of scientific research documents, whether they are published or not. The documents may come from teaching and research institutions in France or abroad, or from public or private research centers.
L'archive ouverte pluridisciplinaire HAL, est destinée au dépôt et à la diffusion de documents scientifiques de niveau recherche, publiés ou non, émanant des établissements d'enseignement et de recherche français ou étrangers, des laboratoires publics ou privés. 


\title{
Self-Regulating Processes
}

\author{
Olivier Barrière*, Antoine Echelard** and Jacques Lévy Véhel**
}

October 8, 2012

\begin{abstract}
We construct functions and stochastic processes for which a functional relation holds between amplitude and local regularity, as measured by the pointwise or local Hölder exponent. We consider in particular functions and processes built by extending Weierstrass function, multifractional Brownian motion and the Lévy construction of Brownian motion. Such processes have recently proved to be relevant models in various applications. The aim of this work is to provide a theoretical background to these studies and to provide a first step in the development of a theory for such self-regulating processes.
\end{abstract}

Keywords. Hölder regularity, Weierstrass function, multifractional Brownian motion, self-regulating processes.

AMS Classification. 60G17, 60G22, 26A16.

${ }^{*}$ Fractales Team, Irccyn, B.P 92101, 44321 Nantes, France

${ }^{* *}$ Regularity Team, Inria, Ecole Centrale Paris - Grande Voie des Vignes, 92295

Châtenay-Malabry Cedex, France

olivier.barriere@gmail.com, antoine.echelard@gmail.com, jacques.levy-vehel@inria.fr

\section{Background and Motivations}

Local regularity of functions and stochastic processes has long been a topic of interest both in Analysis and Probability Theory, with applications in PDE/SPDE, approximation theory or numerical analysis to name a few. Applications outside of mathematics include signal and image processing [4, biomedicine [20] and financial modelling [2]. In many cases, one uses the pointwise or local Hölder exponent to characterize or classify the data. In that view, it is of interest to investigate the construction of functions and processes with everywhere prescribed local regularity. This may be done in various ways, for instance by generalizing Weierstrass function in the deterministic frame (see [11] or Section 2.1), or fractional Brownian motion in a stochastic setting (see [1, 3, 12] or Section 3.1). In this approach, one thus fixes a target regularity function $h$, and builds a function/process whose pointwise Hölder exponent at each point $t$ will be equal to $h(t)$. The local regularity is here set in an exogenous way, in the sense that $h$ is prescribed in an independent manner.

In [16, 17, 18, we have reported on experimental findings indicating that, for certain natural phenomena such as electrocardiograms or natural terrains, there seems to exist a link between the amplitude of the measurements and their pointwise regularity. This intriguing fact prompts for the development of new models, where the regularity would be obtained in an endogenous way: in other words, the Hölder 
exponent at each point would be a function of the value of the process at this point. With such models, one could for instance synthesize numerical terrains which would automatically be more irregular at high altitudes and smooth in valleys.

We define and study in the following functions $f$ and processes $X$ that satisfy a functional relation of the form $\alpha_{f}(t)=g(f(t))$ for all $t$ or $\alpha_{X}(t)=g(X(t))$ almost surely for all $t$, where $\alpha_{f}(t)$ is the pointwise or local Hölder exponent of $f$ at $t$ and $g$ is a smooth deterministic function. A (random) function verifying such a relation will be called self-regulating. In this article, we study three simple examples of such processes: as a warm-up, we consider first a deterministic self-regulating version of generalized Weierstrass function in Section 2. Two constructions of different natures are then presented in a stochastic frame: a self-regulating random process based on multifractional Brownian motion is studied in Section 3, while Section 4 uses a random midpoint displacement technique. Some open problems are presented in Section 5, and appendices in Section 6 gather some of the longer proofs.

Before we begin, we recall for the reader's convenience the definitions of the pointwise and local Hölder exponents.

Definition 1.1. The pointwise Hölder exponent at $x_{0}$ of a continuous function or process $f: \mathbb{R} \rightarrow \mathbb{R}$ is the number $\alpha$ such that:

- $\forall \gamma<\alpha, \lim _{h \rightarrow 0} \frac{\left|f\left(x_{0}+h\right)-P(h)\right|}{|h|^{\gamma}}=0$,

- if $\alpha<+\infty, \forall \gamma>\alpha, \lim \sup _{h \rightarrow 0} \frac{\left|f\left(x_{0}+h\right)-P(h)\right|}{|h|^{\gamma}}=+\infty$

where $P$ is a polynomial of degree not larger than the integer part of $\alpha$.

(This definition is valid only if $\alpha$ is not an integer. It has to be adapted otherwise.)

When $0<\alpha<1$, which will be the main case of interest to us, an equivalent definition reads:

$$
\alpha=\sup \left\{\beta, \limsup _{h \rightarrow 0} \frac{\left|f\left(x_{0}+h\right)-f\left(x_{0}\right)\right|}{|h|^{\beta}}=0\right\} .
$$

Since the pointwise Hölder exponent is defined at each point, one may consider the Hölder function of $f, \alpha_{f}$ : at each $t, \alpha_{f}(t)$ is the pointwise Hölder exponent of $f$ at $t$. When there is no risk of confusion, we shall write $\alpha(t)$ in place of $\alpha_{f}(t)$. Clearly, for $X$ a continuous stochastic process, $\alpha_{X}(t)$ is in general a random variable (with the notable exception of Gaussian processes), so that the pointwise Hölder function is also a stochastic process.

The local Hölder exponent $\alpha_{f}^{l}(t)$ is defined at each point $t$ as the limit when $\rho$ tends to 0 of the global Hölder exponents of $f$ in the ball centred at $t$ with radius $\rho$. Equivalently, for a non-differentiable function $f$,

$$
\alpha_{f}^{l}(t)=\sup \left\{\beta: \exists c, \rho_{0}>0, \forall \rho<\rho_{0}, \sup _{x, y \in B(t, \rho)}|f(x)-f(y)| \leq c|x-y|^{\beta}\right\} .
$$

For simplicity, we will consider functions and processes defined over $[0,1]$ or $[0,1]^{2}$, but the developments below go through without modification to higher dimensions, and, with not much further work, to the case where the domain is the whole of $\mathbb{R}$ or $\mathbb{R}^{n}$. 


\section{Self-Regulating Weierstrass Function}

\subsection{Generalized Weierstrass function}

The celebrated Weierstrass function [21, 22] is defined as follows:

$$
W(t)=\sum_{n=1}^{\infty} \lambda^{-n H} \sin \left(\lambda^{n} t\right),
$$

where $\lambda \geq 2$ and $H>0$. It is well-known that the pointwise and local Hölder exponents of $W$ at each $t$ are equal to $H$. A generalized Weierstrass function of the following form has been considered for instance in [11, 14]:

\section{Definition 2.1.}

Let $h$ be a continuous positive function. The generalized Weierstrass function with functional parameter $h$, denoted $W_{h}$, is:

$$
W_{h}(t)=\sum_{n=1}^{\infty} \lambda^{-n h(t)} \sin \left(\lambda^{n} t\right), \quad \text { where } \lambda \geq 2 .
$$

The pointwise Hölder exponent of $W_{h}$ behaves as follows:

Proposition 2.2 ([1], Proposition 7).

$$
\begin{aligned}
\forall t, & \alpha_{W_{h}}(t) \leq h(t), \\
\text { if } \alpha_{h}(t)>h(t), & \alpha_{W_{h}}(t)=h(t) .
\end{aligned}
$$

\subsection{Self-regulation}

A self-regulating Weierstrass function $S W_{g}$ would be such that $\alpha_{S W_{g}}(t)=g\left(S W_{g}(t)\right)$ at all $t$ for a suitable function $g$. By analogy with the definition of generalized Weierstrass function, one would like to write:

$$
S W_{g}(t)=\sum_{n=1}^{\infty} \lambda^{-n g\left(S W_{g}(t)\right)} \sin \left(\lambda^{n} t\right), \quad \text { where } \lambda \geq 2,
$$

which of course does not provide a valid definition. The usual way to solve an equation as the one above is to use a fixed point approach, and this is the route we shall use. Fix $\alpha>0$, and let $g$ be a $k_{g}$-Lipschitz function from $[0,1]$ to $[\alpha, \infty)$. We shall make use of the following operator:

\section{Definition 2.3.}

Define the map $\Phi$ :

$$
\begin{aligned}
\Phi: \mathcal{C}([0,1],[\alpha,+\infty)) & \rightarrow \mathcal{C}([0,1],[\alpha,+\infty)) \\
h & \mapsto W_{g(h)}+\alpha+\frac{1}{\lambda^{\alpha}-1}
\end{aligned}
$$

where $W_{h}$ is defined as in (3) and $\mathcal{C}(I, J)$ denotes the set of continuous functions from $I$ to $J$. 
Implicit in the definition above is the fact that $\Phi$ does indeed map $\mathcal{C}([0,1],[\alpha,+\infty))$ into itself. This is easily verified: for any $t \in \mathbb{R}$,

$$
\begin{gathered}
-\lambda^{-n \alpha} \leq \lambda^{-n g(h(t))} \sin \left(\lambda^{n} t\right) \leq \lambda^{-n \alpha}, \\
-\sum_{n=1}^{\infty} \lambda^{-n \alpha} \leq W_{g(h)}(t) \leq \sum_{n=1}^{\infty} \lambda^{-n \alpha}=\frac{1}{\lambda^{\alpha}-1},
\end{gathered}
$$

so that:

$$
W_{g(h)}(t)+\alpha+\frac{1}{\lambda^{\alpha}-1} \geq \alpha
$$

\section{Proposition 2.4.}

$\Phi$ possesses a unique fixed point provided $(\alpha, \lambda)$ verify:

$$
k_{\Phi}:=k_{g} \ln (\lambda) \frac{\lambda^{\alpha}}{\left(\lambda^{\alpha}-1\right)^{2}}<1
$$

Proof.

We shall apply Banach fixed point theorem in the space of continuous functions from $[0,1]$ to $[\alpha,+\infty[$, endowed with the sup norm. In that view, we check that $\Phi$ is contractive in this space.

Let $\left(h_{1}, h_{2}\right) \in \mathcal{C}\left([0,1],\left[\alpha,+\infty[)^{2}\right.\right.$. Then, for all $t$ :

$$
\begin{aligned}
\left|\Phi\left(h_{1}(t)\right)-\Phi\left(h_{2}(t)\right)\right| & =\left|\sum_{n=1}^{\infty}\left(\lambda^{-n g\left(h_{1}(t)\right)}-\lambda^{-n g\left(h_{2}(t)\right)}\right) \sin \left(\lambda^{n} t\right)\right| \\
& \leq \sum_{n=1}^{\infty}\left|\lambda^{-n g\left(h_{1}(t)\right)}-\lambda^{-n g\left(h_{2}(t)\right)}\right| .
\end{aligned}
$$

By the finite increments theorem, there exist real numbers $\gamma=\gamma\left(n, g\left(h_{1}(t)\right), g\left(h_{2}(t)\right)\right)$ such that

$$
\lambda^{-n g\left(h_{1}(t)\right)}-\lambda^{-n g\left(h_{2}(t)\right)}=-n \ln (\lambda) \lambda^{-n \gamma}\left(g\left(h_{1}(t)\right)-g\left(h_{2}(t)\right)\right) .
$$

Since all the numbers $\gamma$ are not smaller than $\alpha$, one may write, for all $t$ :

$$
\begin{aligned}
\left|\Phi\left(h_{1}(t)\right)-\Phi\left(h_{2}(t)\right)\right| & \leq \ln (\lambda)\left|g\left(h_{1}(t)\right)-g\left(h_{2}(t)\right)\right| \sum_{n=1}^{\infty} n \lambda^{-n \alpha} \\
& =\ln (\lambda) \frac{\lambda^{\alpha}}{\left(\lambda^{\alpha}-1\right)^{2}}\left|g\left(h_{1}(t)\right)-g\left(h_{2}(t)\right)\right| \\
& \leq k_{g} \ln (\lambda) \frac{\lambda^{\alpha}}{\left(\lambda^{\alpha}-1\right)^{2}}\left|h_{1}(t)-h_{2}(t)\right|,
\end{aligned}
$$

and finally:

$$
\left\|\Phi\left(h_{1}\right)-\Phi\left(h_{2}\right)\right\|_{\infty} \leq k_{g} \ln (\lambda) \frac{\lambda^{\alpha}}{\left(\lambda^{\alpha}-1\right)^{2}}\left\|h_{1}-h_{2}\right\|_{\infty} .
$$

$\Phi$ is thus contractive as soon as $k_{\Phi}<1$.

\section{Theorem 2.1.}

Assume $k_{\Phi}<1$. Let $h^{*}$ denote the fixed point of $\Phi$. Then, for all $t$ :

$$
\alpha_{h^{*}}(t)=g\left(h^{*}(t)\right) .
$$


Proof.

See appendix 6.1

Definition 2.3 has a drawback: it does not allow to control the range of the selfregulated Weierstrass function. It is possible to do so with a slight modification, that will constrain the fixed point to lie in a given interval.

Indeed, consider the map:

$$
\begin{aligned}
\Psi_{\alpha^{\prime}, \beta^{\prime}}: \mathcal{C}([0,1],[\alpha, \beta]) & \rightarrow \mathcal{C}([0,1],[\alpha, \beta]) \\
h & \mapsto \alpha^{\prime}+\left(\beta^{\prime}-\alpha^{\prime}\right){\underset{(t, H) \in[0,1] \times[\alpha, \beta]}{\max _{(t, H) \in[0,1] \times[\alpha, \beta]}\left(W_{H}(t)\right)-} \min _{(t, H) \in[0,1] \times[\alpha, \beta]}\left(W_{H}(t)\right)}_{\left.W_{H}(t)\right)}
\end{aligned}
$$

where $\alpha, \alpha^{\prime}, \beta$ and $\beta^{\prime}$ such that $0<\alpha \leq \alpha^{\prime}<\beta^{\prime} \leq \beta$.

It is easily proved that $\Psi_{\alpha^{\prime}, \beta^{\prime}}$ does map $\mathcal{C}([0,1],[\alpha, \beta])$ into itself.

We leave it to the reader to show the following extension of Proposition 2.4

\section{Proposition 2.5.}

The map $\Psi_{\alpha^{\prime}, \beta^{\prime}}$ possesses a unique fixed point provided $\left(\alpha^{\prime}, \beta^{\prime}, \lambda\right)$ verify:

$$
\frac{\left(\beta^{\prime}-\alpha^{\prime}\right) k_{g} \ln (\lambda) \lambda^{\alpha}}{\left(\max \left(W_{\alpha}\right)-\min \left(W_{\alpha}\right)\right)\left(\lambda^{\alpha}-1\right)^{2}}<1 .
$$

One may obtain self-regulating Weierstrass functions by starting for instance from the constant function equal to $(\alpha+\beta) / 2$ and iterating $\Psi_{\alpha^{\prime}, \beta^{\prime}}$.

Figure 1 displays the graph of such a function sampled on 65536 points. Also shown is the estimated regularity using an oscillation-based approach. Both the synthesis and estimation methods are available in the FracLab software toolbox [15].

\section{Self-Regulating Multifractional Brownian Mo- tion}

In this and the next section, we shall consider stochastic versions of the selfregulating property. Our first construction is based on multifractional Brownian motion. We briefly recall below some basic facts about fractional and multifractional Brownian motions.

\subsection{Background on multifractional Brownian motion}

\subsubsection{Fractional and multifractional Brownian motions}

Fractional Brownian motion (fBm) [7, 10] is a centred Gaussian process with features that make it a useful model in various applications such as financial and teletraffic 


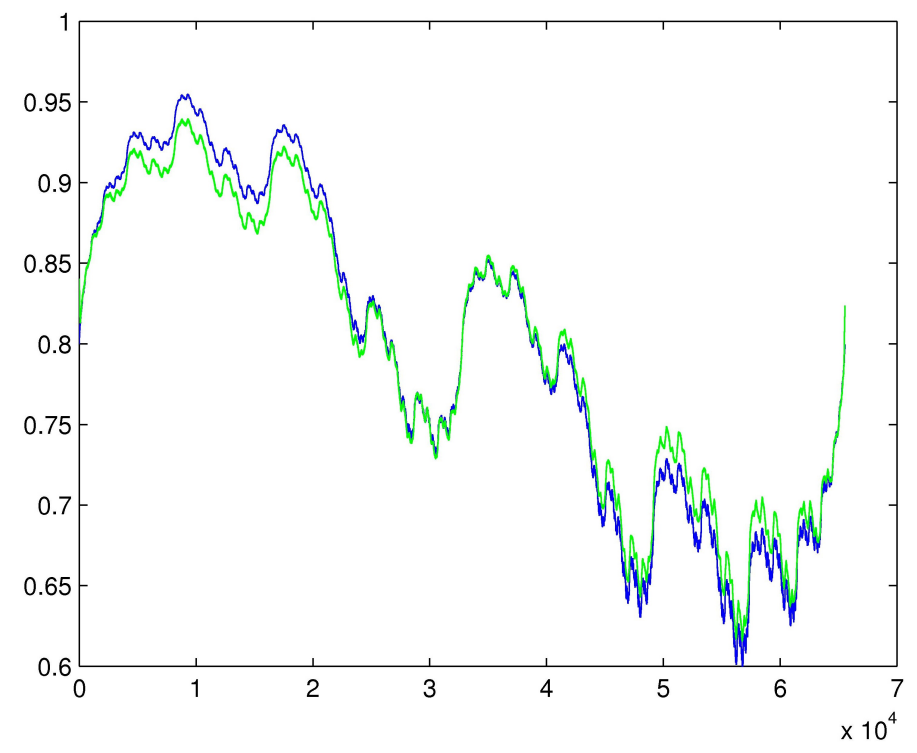

Figure 1: Self-regulating Weierstrass function with $g(x)=x, \lambda=2, \alpha=0.6, \beta=1$ (blue) and estimated exponents (green). Notice that the graph is smoother where it takes larger values and vice-versa.

modeling, image analysis and synthesis, geophysics and more. These features include self-similarity, long-range dependence and the ability to match any prescribed constant local regularity. Fractional Brownian motion depends on a parameter, usually denoted by $H$ and called the Hurst exponent, that belongs to $(0,1)$. Its covariance function $R_{H}$ reads:

$$
R_{H}(t, s):=\frac{\gamma_{H}}{2}\left(|t|^{2 H}+|s|^{2 H}-|t-s|^{2 H}\right),
$$

where $\gamma_{H}$ is a positive constant. When $H=\frac{1}{2}$, fBm reduces to Brownian motion. While $\mathrm{fBm}$ is a useful model, the fact that most of its properties are governed by the single number $H$ restricts its application in some situations. In particular, its Hölder exponent remains the same all along its trajectory. Thus, for instance, fBm with long range dependent increments, which require $H>\frac{1}{2}$, must have smoother paths than Brownian motion. Multifractional Brownian motion [12, 3] was introduced to overcome these limitations. The basic idea is to replace the real $H$ by a function $t \mapsto h(t)$ ranging in $(0,1)$.

The construction of $\mathrm{mBm}$ is best understood through the use of a fractional Brownian field [8]. Fix a probability space $(\Omega, \mathcal{F}, P)$. A fractional Brownian field on $(0,1) \times[0,1]$ is a Gaussian field, denoted $(B(H, t))_{(H, t) \in(0,1) \times[0,1]}$, such that for every $H$ in $(0,1)$ the process $\left(B_{t}^{H}\right)_{t \in[0,1]}$, where $B_{t}^{H}:=B(H, t)$, is a fractional Brownian motion with parameter $H$. For a deterministic continuous function $h:[0,1] \rightarrow(0,1)$, a multifractional Brownian motion with functional parameter $h$ is the Gaussian process $B^{h}:=\left(B_{t}^{h}\right)_{t \in[0,1]}$ defined by $B_{t}^{h}:=B(h(t), t)$. The function $h$ is called the regularity function of $\mathrm{mBm}$.

The class of $\mathrm{mBm}$ is rather large, since there is some freedom in choosing the correlations between the fBms composing the fractional field $B(t, H)$. For definiteness, we will consider in the sequel the so-called "well-balanced" version of multifractional Brownian motion. Essentially the same analysis could be conducted with 
other versions. More precisely, a well-balanced $\mathrm{mBm}$ is obtained from the field $B(H, t):=\frac{1}{c_{H}} \int_{\mathbb{R}} \frac{e^{i t u}-1}{|u|^{H+1 / 2}} \widetilde{W}(d u)$ where $\widetilde{W}$ denotes a complex-valued Gaussian measure (cf. [13 for more details). Its covariance $R_{h}$ reads:

$$
R_{h}(t, s)=\frac{c_{h_{t, s}}^{2}}{c_{h(t)} c_{h(s)}}\left(\frac{1}{2}\left(|t|^{2 h_{t, s}}+|s|^{2 h_{t, s}}-|t-s|^{2 h_{t, s}}\right)\right),
$$

where $h_{t, s}:=\frac{h(t)+h(s)}{2}$ and $c_{x}:=\left(\frac{2 \pi}{\Gamma(2 x+1) \sin (\pi x)}\right)^{\frac{1}{2}}$.

The other main properties of $\mathrm{mBm}$ are the following ones: the pointwise Hölder exponent at any point $t$ of $B^{h}$ is almost surely equal to $h(t) \wedge \alpha_{h}(t)$. For a smooth $h$ (e.g. $\left.C^{1}\right)$, the equality is known to hold in a uniform sense, that is, $\alpha(t)=h(t)$ almost surely for all $t$. One may thus control the local regularity of the paths through the evolution of $h$. In addition, the increments of $\mathrm{mBm}$ display long range dependence for all non-constant $h(t)$ (long range dependence must be defined in an adequate way since the increments are not stationary. See [9] for more details). Finally, when $h$ is $C^{1}, \mathrm{mBm}$ is tangent to $\mathrm{fBm}$ with exponent $h(u)$ in the neighbourhood of any $u$ in the following sense [5]:

$$
\lim _{r \rightarrow 0_{+}} \frac{B^{h}(u+r t)-B^{h}(u)}{r^{h(u)}}=B^{h}(t),
$$

where the convergence holds in law. This is essentially a consequence of the fact that the field $B(H, t)$ is smooth in the $H$ variable (see below for a precise statement).

These properties show that $\mathrm{mBm}$ is a more versatile model that $\mathrm{fBm}$ : in particular, it is able to mimic in a more faithful way local properties of financial records, Internet traffic and natural landscapes by matching their local regularity. See http://regularity.saclay.inria.fr/theory/stochasticmodels/bibliombm for a sample of applications of mbm.

\subsubsection{Multifractional Process with Random Exponent}

It seems natural to generalize the definition of $\mathrm{mBm}$ to include the case where $h$ is no longer deterministic but random. Of course, if $h$ is independent of the field $B$, this raises no difficulty. The interesting situation is when the two processes may be correlated. This case was studied in [8], where the resulting process is termed Multifractional Process with Random Exponent (MPRE).

To define such a process, one starts from a field $\{B(H, t)\}$ and a stochastic process $\{S(t)\}_{t \in[0,1]}$ with values in $[a, b] \subset(0,1)$. The MPRE $\{X(t)\}_{t \in[0,1]}$ is then defined by:

$$
X(t, \omega)=B(S(t, \omega), t, \omega) .
$$

In [8], a condition is imposed throughout on the global Hölder exponent $\beta_{S}([0,1])$ of $S$ over [0,1]. Namely, with probability 1 :

$$
\sup _{t \in[0,1]} S(t, \omega)<\beta_{S}([0,1], \omega) .
$$

Under this assumption, one of the main results of $[8]$ is that, for any $t \in[0,1]$, one has almost surely:

$$
\alpha_{X}(t, \omega)=S(t, \omega) .
$$

We will see that, in our case, (11) cannot possibly hold, so that we will have to extend the result above in order to obtain the self-regulating property.

The following properties of the field $\{B(H, t)\}_{H, t \in[a, b] \times[0,1]}$ will be needed: 
Proposition 3.1 ([8], Proposition 2.2). There is an event $\Omega_{1}^{*}$ of probability 1 such that, for any $\omega \in \Omega_{1}^{*}$,

- the function $(H, t) \mapsto B(H, t, \omega)$ is continuous over $[a, b] \times[0,1]$,

- for all reals $m$ and $M$ such as $a \leq m \leq M \leq b$, the uniform Hölder exponent of the function $(H, t) \mapsto B(H, t, \omega)$ over the rectangle $[m, M] \times[0,1]$ is equal to $m$ : in other words, for any $\epsilon>0$, there is a random variable $C_{1}$ which only depends on $m, M$ and $\epsilon$ such what the following inequality holds for all $\omega \in \Omega_{1}^{*},\left(t, t^{\prime}, H, H^{\prime}\right) \in[0,1]^{2} \times[m, M]^{2}$ :

$$
\left|B(H, t, \omega)-B\left(H^{\prime}, t^{\prime}, \omega\right)\right| \leq C_{1}(\omega)\left(\left|t-t^{\prime}\right|+\left|H-H^{\prime}\right|\right)^{m-\varepsilon},
$$

- for any $t \in[0,1]$, the function $H \mapsto B(H, t, \omega)$ is continuously differentiable over $[a, b]$,

- the function $H \mapsto B(H, t, \omega)$ is Lipschitz over $[a, b]$ uniformly in $t \in[0,1]$. More precisely, there exists an almost surely finite random variable $C_{2}$ (which only depends on $a$ and $b)$ such as for every $\left(H, H^{\prime}\right) \in[a, b]^{2}$ one has

$$
\sup _{t \in[0,1]}\left|B(H, t, \omega)-B\left(H^{\prime}, t, \omega\right)\right| \leq C_{2}(\omega)\left|H-H^{\prime}\right|
$$

where

$$
C_{2}(\omega)=\sup _{(H, t) \in[a, b] \times[0,1]}\left|\left(\frac{\partial}{\partial H}\right) B(H, t, \omega)\right| .
$$

\subsection{Self-regulating $\mathrm{mBm}$}

We now come to self-regulating versions of $\mathrm{mBm}$ (srmBm for short). In other words, we wish to define a process $Z=Z(g)$ such that, at each point, almost surely:

$$
\alpha_{Z}(t, \omega)=g(Z(t, \omega))
$$

where $g$ is a deterministic $k_{g}$-Lipschitz function defined on an interval $[\alpha, \beta] \subset \mathbb{R}$ and ranging in $[a, b] \subset(0,1)$.

We shall present two constructions of srmBm. The first one is based on a fixed point approach, while the second one is geometrical.

Before we begin, we mention that the technique used in subsubsection 3.2.1 generalizes with a few additional technicalities to construct "self-stabilizing" processes, i.e. processes where the local index of stability varies in time (see e.g. [6]): it suffices to replace the fractional Brownian field $B$ by a stable field and to use an adequate metric. This will be developed in a forthcoming work. In addition, it does not cost anything to replace the deterministic $g$ by a random function of the form $g=g(t, \omega)$, so that the self-regulating relation takes the form $\alpha_{Z}(t, \omega)=g(Z(t, \omega), \omega)$, as long as the (random) Lipschitz constant is such that $k_{g}(\omega)<k$ for all $\omega$, where $k$ is a fixed real number.

\subsubsection{Fixed point srmBm}

The following notation will prove useful:

\section{Definition 3.2.}


Let $X$ be a continuous non constant field defined on a compact set $K$, and let $\alpha^{\prime}, \beta^{\prime}$ be two real numbers. Denote $\underline{\bar{X}}_{\alpha^{\prime}}^{\beta^{\prime}}$ the scaled field:

$$
\underline{X}_{\alpha^{\prime}}^{\beta^{\prime}}=\alpha^{\prime}+\left(\beta^{\prime}-\alpha^{\prime}\right) \frac{X-\min _{K}(X)}{\max _{K}(X)-\min _{K}(X)} .
$$

We consider the following stochastic operator:

\section{Definition 3.3.}

Let $\alpha^{\prime}(\omega), \beta^{\prime}(\omega)$ be two random variables such that $\alpha \leq \alpha^{\prime}(\omega)<\beta^{\prime}(\omega) \leq \beta$. The stochastic operator $\Lambda_{\alpha^{\prime}, \beta^{\prime}}$ is defined for all $\omega \in \Omega_{1}^{*}$ as:

$$
\begin{aligned}
\Lambda_{\alpha^{\prime}, \beta^{\prime}}: \mathcal{C}([0,1],[\alpha, \beta]) & \rightarrow \mathcal{C}([0,1],[\alpha, \beta]) \\
Z & \mapsto \underline{B g(Z)}^{B_{g}(\omega)} \alpha^{\prime}
\end{aligned}
$$

where $B_{g(Z)}(\omega)$ denotes the function $t \mapsto B_{g(Z(t))}(t, \omega)$.

It is easy to check that $\Lambda_{\alpha^{\prime}, \beta^{\prime}}$ is well-defined and measurable.

\section{Proposition 3.4.}

$\Lambda_{\alpha^{\prime}, \beta^{\prime}}$ possesses a unique fixed point provided condition $\mathcal{C}$ holds:

$\mathcal{C}: \quad \beta^{\prime}(\omega)-\alpha^{\prime}(\omega)<\frac{\max _{(t, H) \in[0,1] \times[a, b]}\left(B_{H}(t, \omega)\right)-\min _{(t, H) \in[0,1] \times[a, b]}\left(B_{H}(t, \omega)\right)}{C_{2}(\omega) k_{g}}$.

We shall denote $Z_{g}$ this fixed point:

$$
Z_{g}(\omega)=\underline{\overline{B_{g\left(Z_{g}(\omega)\right)}(\omega)}}{ }^{\beta^{\prime}(\omega)} \alpha^{\prime}(\omega)
$$

and call it fixed point srmBm.

Note that, by construction, $Z_{g}$ ranges in $[\alpha, \beta]$. By choosing adequately the interval of definition of $g$, one may control the values taken by the process.

Proof.

We show that $\Lambda_{\alpha^{\prime}, \beta^{\prime}}$ is contractive in the set of continuous functions from $[0,1]$ to $[\alpha, \beta]$ equipped with the sup norm.

Let:

$$
\begin{aligned}
& M_{B}(\omega)=\max _{(t, H) \in[0,1] \times[a, b]}\left(B_{H}(t, \omega)\right), \\
& m_{B}(\omega)=\min _{(t, H) \in[0,1] \times[a, b]}\left(B_{H}(t, \omega)\right) .
\end{aligned}
$$

By definition, for all $Z$ in $\mathcal{C}([0,1],[\alpha, \beta])$ :

$$
\begin{aligned}
\forall t \in[0,1], \Lambda_{\alpha^{\prime}, \beta^{\prime}}(Z(t)) & =\overline{B_{g(Z(t))}(t, \omega)} \beta^{\prime} \\
& =\alpha^{\prime}{ }^{\prime}+\left(\beta^{\prime}-\alpha^{\prime}\right) \frac{B_{g(Z(t))}(t, \omega)-m_{B}(\omega)}{M_{B}(\omega)-m_{B}(\omega)} .
\end{aligned}
$$

As a consequence, for $\left(Z_{1}, Z_{2}\right)$ in $\mathcal{C}([0,1],[\alpha, \beta])^{2}$ and for all $t$ in $[0,1]$ :

$\Lambda_{\alpha^{\prime}, \beta^{\prime}}\left(Z_{1}(t)\right)-\Lambda_{\alpha^{\prime}, \beta^{\prime}}\left(Z_{2}(t)\right)=\frac{\beta^{\prime}-\alpha^{\prime}}{M_{B}(\omega)-m_{B}(\omega)}\left(B_{g\left(Z_{1}(t)\right)}(t, \omega)-B_{g\left(Z_{2}(t)\right)}(t, \omega)\right)$. 
Inequality (13) and the Lipschitz property of $g$ entail:

$$
\begin{aligned}
\left|\Lambda_{\alpha^{\prime}, \beta^{\prime}}\left(Z_{1}(t)\right)-\Lambda_{\alpha^{\prime}, \beta^{\prime}}\left(Z_{2}(t)\right)\right| & \leq \frac{\beta^{\prime}-\alpha^{\prime}}{M_{B}(\omega)-m_{B}(\omega)} C_{2}(\omega)\left|g\left(Z_{1}(t)\right)-g\left(Z_{2}(t)\right)\right| \\
& \leq \frac{\left(\beta^{\prime}-\alpha^{\prime}\right) C_{2}(\omega)}{M_{B}(\omega)-m_{B}(\omega)} k_{g}\left|Z_{1}(t)-Z_{2}(t)\right| .
\end{aligned}
$$

Thus:

$$
\left\|\Lambda_{\alpha^{\prime}, \beta^{\prime}}\left(Z_{1}\right)-\Lambda_{\alpha^{\prime}, \beta^{\prime}}\left(Z_{2}\right)\right\|_{\infty} \leq \frac{\left(\beta^{\prime}-\alpha^{\prime}\right) C_{2}(\omega) k_{g}}{M_{B}(\omega)-m_{B}(\omega)}\left\|Z_{1}-Z_{2}\right\|_{\infty}
$$

which shows that $\Lambda_{\alpha^{\prime}, \beta^{\prime}}$ is contractive under condition $\mathcal{C}$.

Remark 3.5. Note that, when $g$ is constant and equal to $H$, srmBm is just a scaled $f B m$.

We need to prove that $Z_{g}$ is indeed self-regulating:

\section{Theorem 3.1.}

For all $t$ in $[0,1]$, almost surely :

$$
\alpha_{Z_{g}}(t, \omega)=g\left(Z_{g}(t, \omega)\right)
$$

Proof.

See Appendix 6.2

Corollary 3.6. Assume $g$ is not constant. Then $Z_{g}$ is not a Gaussian process.

Proof. For a Gaussian process $X$, the Hölder exponent of $X$ at each point assumes an almost sure value. Except in the trivial case where $g$ is constant, this cannot be the case for an srmBm $Z_{g}$.

Remark 3.7. A representation equivalent (in law) to the one we have used for $m B m$ reads:

$$
B^{h}(t)=\int_{\mathbb{R}}\left(|t-u|^{h(t)-1 / 2}-|u|^{h(t)-1 / 2}\right) W(d u) .
$$

Now that one knows that $Z_{g}$ exists, one may be tempted to write:

$$
Z_{g}(t)=\alpha^{\prime}+\frac{\beta^{\prime}-\alpha^{\prime}}{M_{B}-m_{B}}\left(\int_{\mathbb{R}}\left(|t-u|^{g\left(Z_{g}(t)\right)-1 / 2}-|u|^{g\left(Z_{g}(t)\right)-1 / 2}\right) W(d u)-m_{B}\right) .
$$

However, the integrand is not adapted to the filtration generated by $W$. The integral is thus not defined as an Itô integral. It may nevertheless exist in the more general Wick-Itô sense. We shall address this question in a forthcoming paper.

The rescaling of the fractional Brownian field $B(H, t)$ is a necessary step to ensure uniqueness of $Z_{g}$. Unfortunately, it severely complicates the analysis: computing the law of $\left(\underline{\underline{B_{H}(t, \omega)}} \alpha^{\beta^{\prime}}\right)$ seems currently out of reach. We only provide an extremely simple result in this direction: 


\section{Proposition 3.8.}

Let $\bar{\alpha}<\bar{\beta}$ be two real numbers. Then,

$$
\forall(t, H) \in[0,1] \times[a, b], \mathbb{E}\left({\overline{B_{H}(t, \omega)}}_{\bar{\alpha}}^{\bar{\beta}}\right)=\frac{\bar{\alpha}+\bar{\beta}}{2} .
$$

Proof.

Note first that

$$
{\overline{B_{H}(t, \omega)}}_{\bar{\alpha}}^{\bar{\beta}}=\bar{\alpha}+(\bar{\beta}-\bar{\alpha}){\underline{\overline{B H}_{H}(t, \omega)}}_{0}^{1} .
$$

Thus, it suffices to prove the result with $\bar{\alpha}=0, \bar{\beta}=1$.

Let $\widetilde{B_{H}(t)}$ denote the reflected fractional Brownian field $\widetilde{B_{H}(t)}=-B_{H}(t)$. For any fixed $t, \widetilde{B_{H}(t)}$ and $B_{H}(t)$ have same distribution. In addition, writing $\widetilde{m_{B}}=\min \left(\widetilde{B_{H}(t)}\right)$ and $\widetilde{M_{B}}=\max \left(\widetilde{B_{H}(t)}\right)$, one has

$$
\widetilde{m_{B}}=-M_{B} \text { and } \widetilde{M_{B}}=-m_{B}
$$

Thus the following equalities hold in law:

$$
\begin{aligned}
\frac{B_{H}(t)-m_{B}}{M_{B}-m_{B}} & =\frac{\widetilde{B_{H}(t)}-\widetilde{m_{B}}}{\widetilde{M_{B}}-\widetilde{m_{B}}} \\
& =\frac{-B_{H}(t)+M_{B}}{-m_{B}+M_{B}} \\
& =1-\left(\frac{B_{H}(t)-m_{B}}{M_{B}-m_{B}}\right) .
\end{aligned}
$$

As a consequence:

$$
\mathbb{E}\left({\underline{\bar{B}_{H}(t)}}_{0}^{1}\right)=1-\mathbb{E}\left({\underline{\bar{B}_{H}(t)}}_{0}^{1}\right) .
$$

One may obtain paths of srmBm by using the fixed point property: one first synthesizes a fractional Brownian field and rescale it appropriately. Then, starting from an arbitrary $H$ value and a corresponding "line" on the field, one computes iterates $Z_{n+1}=\Lambda_{\alpha^{\prime}, \beta^{\prime}}\left(Z_{n}\right)=\overline{B_{g\left(Z_{n}\right)}(\omega)}{ }_{\alpha^{\prime}}{ }^{\prime}$. This sequence will (almost surely) converge to $Z_{g}$. Iterations are stopped as usual when the sup norm of the difference $Z_{n+1}-Z_{n}$ falls below a certain threshold. We show in Figure 2 some examples of srmBm obtained in this way using FracLab. One clearly see how regularity relates to amplitude. For instance, in the top right plot, the path is more irregular when the process has values close to $\frac{1}{2}$ and is smoother when its amplitude is close to 0 or 1 .

Let us discuss briefly some features of the above simulation algorithm. Two possible sources of error occur: the first one lies in computing the fractional Brownian field, while the second one comes from the iteration procedure. Let us consider the first source of error. There is a vast literature on the simulation of $\mathrm{fBm}$. As this is a Gaussian process, an exact method is provided by the use of a Cholesky decomposition. Such a method is slow in general. However, in the case of fBm, 

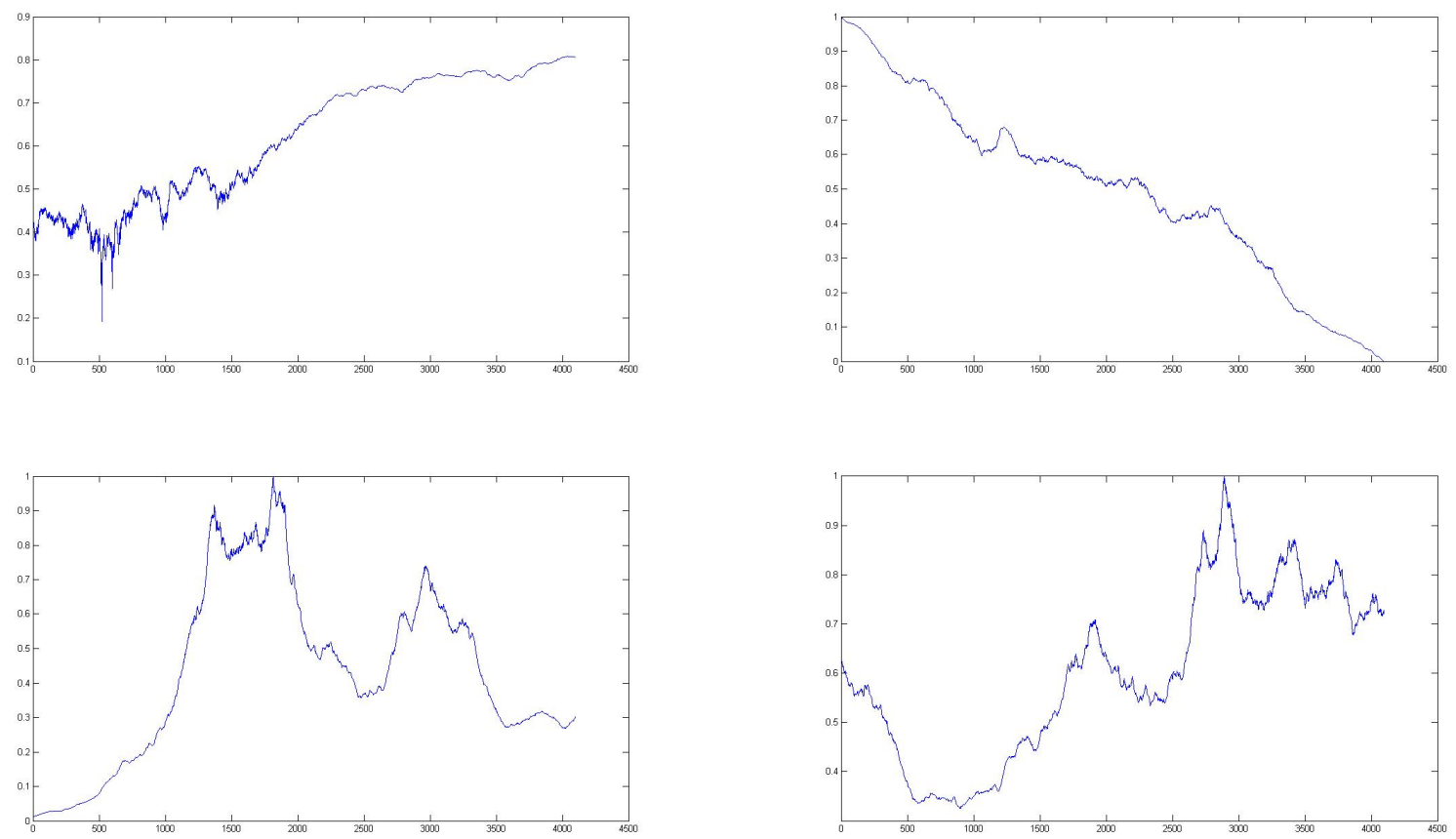

Figure 2: Top left: srmBm with $\alpha(t)=Z(t)$. Top right: $\operatorname{srmBm}$ with $\alpha(t)=0.6+$ $(|Z(t)|-0.5)^{2}$. Bottom: two srmBm with $\alpha(t)=\frac{1}{1+\frac{|Z(t)|}{2}}$.

one may take advantage of the stationarity of the increments and use the algorithm described in [23] to obtain exact (within numerical precision) paths of $\mathrm{fBm}$ with a cost of $\mathcal{O}(N \log (N))$, where $N$ is the number of samples. To obtain our fractional Brownian field, we generate $M$ fBms with this method with values $\left(H_{i}\right)_{i=1, \ldots, M}$ regularly spaced between $a$ and $b$. During the iterations of the process yielding srmBm, we shall need the value of the field at arbitrary $H$. We will approximate this value by the one of the computed field at $H_{i}(H)$, where $H_{i}(H)$ is the value in $\left\{H_{i}, i=1, \ldots, M\right\}$ which is closest to $H$. The difference between $H$ and $H_{i}(H)$ is at most $(b-a) / 2 M$. By smoothness of the field in the $H$ direction, the error on the corresponding point on the field is of the same order, almost surely and uniformly in $t$ on any compact. It is important to notice that such errors do not propagate through iterations: indeed, at step $n$, instead of working with the exact $Z_{n}$, we deal with an approximate one, say $\tilde{Z}_{n}$. However, when the stopping criterion is met, we do have $\left\|\tilde{Z}_{n+1}-\tilde{Z}_{n}\right\|<\varepsilon$, where $\varepsilon$ is the threshold. As a consequence, the difference in sup norm between $Z$ and $\tilde{Z}_{n}$ is at most $C(\varepsilon+(b-a) / M)$ for a constant $C$. This is the precision of our algorithm. Its cost is is $\mathcal{O}(P N+M N \log (N))$, where $P$ is the number of iterations. While we do not have a theoretical bound on $P$, numerical experiments show that it is usually negligible as compared to both $M$ and $\log (N)$.

Though all the developments above were conducted in one dimension, the extension to $\mathbb{R}^{n}$ is straightforward. Figure 3 displays an example of a two-dimensional srmBm with $g(Z)=(1-Z)^{2}$. This particular choice of $g$ is adapted to natural terrain modelling and reflects the fact, in young mountains, regions at higher altitudes are typically more irregular than ones at low altitude. 


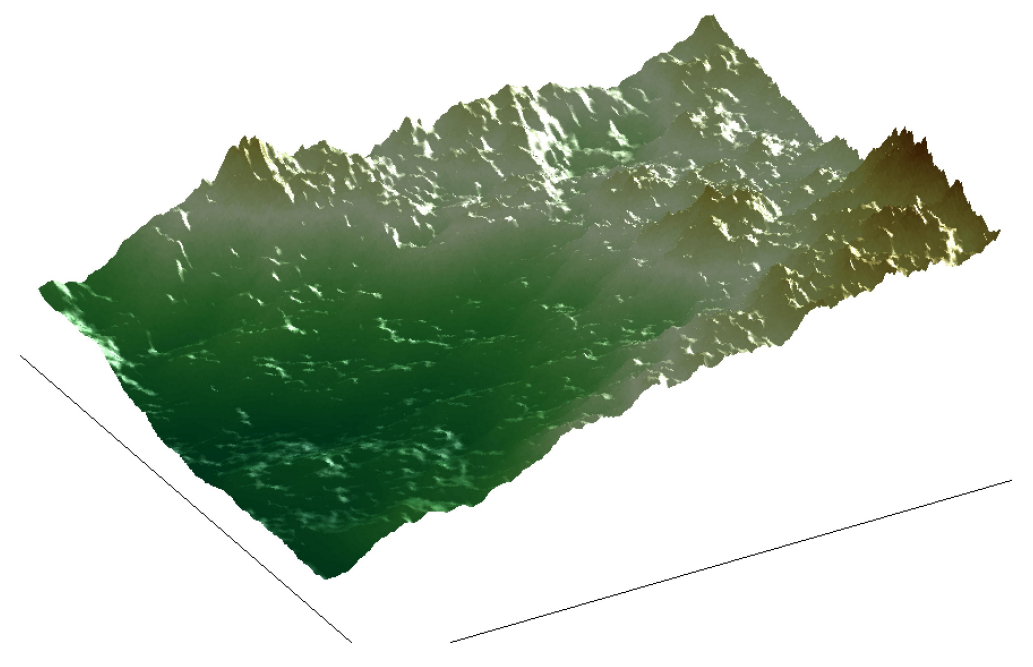

Figure 3: A two-dimensional srmBm with $g(Z)=(1-Z)^{2}$.

\subsubsection{Geometrical srmBm}

When the function $g$ is smooth, a simple geometrical reasoning allows to build a self-regulating process on a fractional Brownian field. The idea is that, in the three-dimensional space $(t, H, B)$, an srmBm is the intersection of the two surfaces

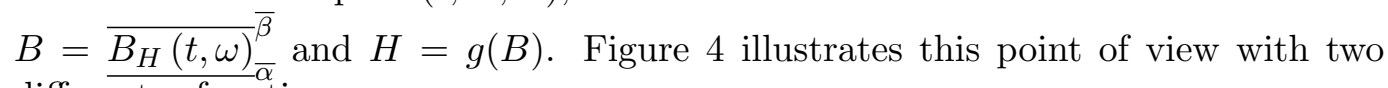
different $g$ functions.

For simplicity, we shall assume that $g$ is a diffeomorphism and will denote $f=g^{-1}$.

\section{Definition 3.9.}

Let $\alpha^{\prime}, \beta^{\prime}$ be real numbers with $\alpha \leq \alpha^{\prime}<\beta^{\prime} \leq \beta$. Define:

$$
\begin{aligned}
\Theta_{\alpha^{\prime}, \beta^{\prime}}:[0,1] \times[\alpha, \beta] & \rightarrow\left[\alpha^{\prime}-\beta, \beta^{\prime}-\alpha\right] \\
(t, U) & \mapsto \frac{B_{g(U)}(t, \omega)}{\beta^{\prime}} \alpha^{\prime}-U .
\end{aligned}
$$

\section{Proposition 3.10.}

For almost all $\omega$ and all $t$, there exists at least one value $Z^{\times}(t, \omega)$ such that:

$$
Z^{\times}(t, \omega)=\underline{\overline{B_{g\left(Z^{\times}(t, \omega)\right)}(t, \omega)}}{ }_{\alpha^{\prime}}^{\beta^{\prime}} .
$$

Proof. Note first that $\Theta_{\alpha^{\prime}, \beta^{\prime}}$ is continuous (it is in fact Hölder continuous. Indeed:

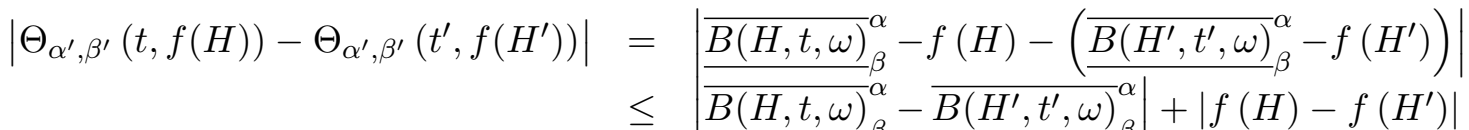

$$
\begin{aligned}
& \left.\leq C_{1}(\omega) \frac{\beta-\alpha}{M_{B}-m_{B}}\left(\left|t-t^{\prime}\right|+\left|H-H^{\prime}\right|\right)^{m-\varepsilon}+\left|f(H)-f\left(H^{\prime}\right)\right| .\right)
\end{aligned}
$$

Therefore, every level set of $\Theta_{\alpha^{\prime}, \beta^{\prime}}$ is itself continuous.

In addition, $\Theta_{\alpha^{\prime}, \beta^{\prime}}$ verifies almost surely: 

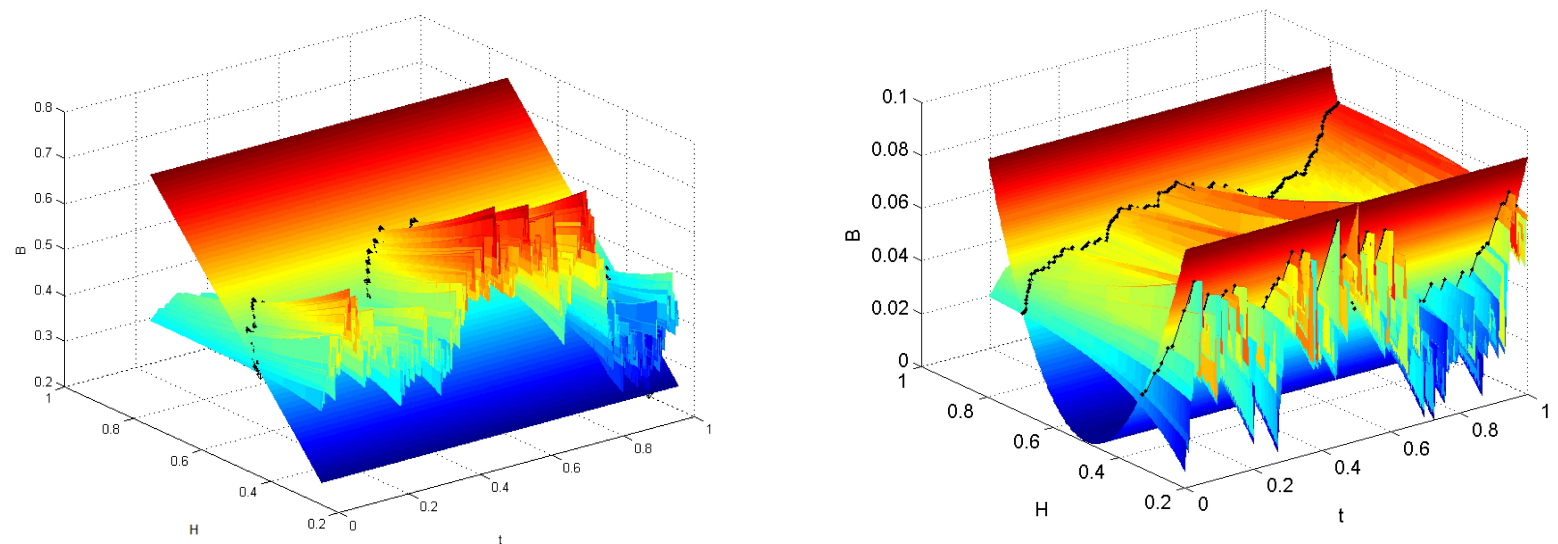

Figure 4: Intersections between a scaled fractional Brownian field and two surfaces $H=$ $g(B)$. Note that, when $g$ is not one to one as on the figure on the right, several srmBms with different regularity will be obtained.

- $\forall t \in[0,1], \Theta_{\alpha^{\prime}, \beta^{\prime}}(t, \alpha)=\underline{\overline{B g(\alpha)}_{(t, \omega)}}{ }^{\beta^{\prime}} \alpha^{\prime}-\alpha \geq 0$,

- $\forall t \in[0,1], \Theta_{\alpha^{\prime}, \beta^{\prime}}(t, \beta)={\overline{B_{g(\beta)}(t, \omega)}}_{\alpha^{\prime}}^{\beta^{\prime}}-\beta \leq 0$.

The intermediate value theorem then entails that, for all $t$, there exists $Z^{\times}(t)$ such that $\Theta_{\alpha^{\prime}, \beta^{\prime}}\left(t, Z^{\times}(t)\right)=0$.

\section{Proposition 3.11.}

Let $\mu=\sup _{U \in[\alpha, \beta]}\left|g^{\prime}(U)\right|$. Let $\alpha^{\prime}(\omega)$ and $\beta^{\prime}(\omega)$ be such that $\alpha<\alpha^{\prime}(\omega)<\beta^{\prime}(\omega)<\beta$ and $\left(\alpha^{\prime}(\omega), \beta^{\prime}(\omega)\right)$ verify Condition $\mathcal{C}_{2}$ :

$$
\mathcal{C}_{2}: \quad \beta^{\prime}(\omega)-\alpha^{\prime}(\omega)<\frac{M_{B}(\omega)-m_{B}(\omega)}{\mu C_{2}(\omega)} .
$$

Then, almost surely, there exits a unique function $Z^{\times}(t)$ such that:

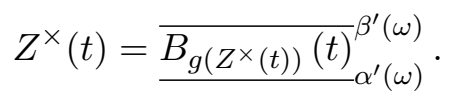

Proof.

One computes:

$$
\begin{aligned}
\frac{\partial \Theta}{\partial U}(t, U) & =\frac{\partial \overline{B_{g(U)}(t, \omega)}{ }^{\beta^{\prime}}}{\partial U}-1 \\
& =\frac{\beta^{\prime}-\alpha^{\prime}}{M_{B}(\omega)-m_{B}(\omega)} \frac{\partial B_{g(U)}(t, \omega)}{\partial U}-1 \\
& =\left.\frac{\beta^{\prime}-\alpha^{\prime}}{M_{B}(\omega)-m_{B}(\omega)} g^{\prime}(U) \frac{\partial B_{H}(t, \omega)}{\partial H}\right|_{H=g(U)}-1 .
\end{aligned}
$$

Assumption $\mathcal{C}_{2}$ entails that $U \mapsto \Theta_{\alpha^{\prime}(\omega), \beta^{\prime}(\omega)}(t, U)$ is decreasing. 
Proposition 3.11 yields an algorithm to generate a geometrical srmBm: one first synthesize a fractional Brownian field $B$ as explained in Subsection 3.2.1, and then numerically compute its interesction with the surface $H=g(B)$. The computational cost is $\mathcal{O}(M N \log (N))$ (with the same notations as above). Errors occur when evaluating the values of $B$ at non-sampled values of $H$, as in Subsetion 3.2.1, and also when evaluating the values of $g(B)$. Since $g$ is smooth, these errors are of the same order as the ones on $B$ itself. As a consequence, the precision of the whole algorithm is of order $(b-a) / M$.

\subsection{Prescribed shape srmBm}

The fixed point srmBm of section 3.2.1 only depends on $g$. Thus, if, say, $g(Z)=Z$, one may have a particular realization which is smooth because the values of $Z$ will be large, while another one will appear irregular if it happens that $Z$ takes only small values. This may be a drawback in certain applications. We briefly describe in this section how to modify the definition of fixed point srmBm so that it allows to follow a prescribed overall trend.

\section{Definition 3.12.}

Let $s$ be a $C^{1}$ function from $[0,1]$ to $\mathbb{R}$, and $m$ be a positive real number. Define the Gaussian field $\left\{B_{H}^{(s)}(t)\right\}_{(H, t) \in[a, b] \times[0,1]}$ as:

$$
B_{H}^{(s)}(t, \omega)=\frac{\sup _{t \in[0,1]}(s(t))}{B_{H}(t, \omega)} \inf _{t \in[0,1]}(s(t))+m s(t) .
$$

Since $s$ is smooth, the regularity properties of $B_{H}^{(s)}(t)$ are similar to the ones of $B_{H}(t)$. In addition, it is straightforward to check that, for $\alpha^{\prime}(\omega)$ and $\beta^{\prime}(\omega)$ two random variables such that $\alpha \leq \alpha^{\prime}(\omega)<\beta^{\prime}(\omega) \leq \beta$, the operator $\Lambda_{\alpha^{\prime}, \beta^{\prime}}^{(s)}$ defined for almost all $\omega$ by:

$$
\begin{aligned}
\Lambda_{\alpha^{\prime}, \beta^{\prime}}^{(s)}: \mathcal{C}([0,1],[\alpha, \beta]) & \rightarrow \mathcal{C}([0,1],[\alpha, \beta]) \\
Z & \mapsto \underline{B g(Z)}(\omega)_{\alpha^{\prime}}^{(s)} \alpha^{\prime}
\end{aligned}
$$

is contractive provided:

$\mathcal{H}^{(s)}: \quad \beta^{\prime(s)}(\omega)-\alpha^{\prime(s)}(\omega)<\frac{\max _{(t, H) \in[0,1] \times[a, b]}\left(B_{H}^{(s)}(t, \omega)\right)-\min _{(t, H) \in[0,1] \times[a, b]}\left(B_{H}^{(s)}(t, \omega)\right)}{C_{2}^{(s)}(\omega) k_{g}}$.

The prescribed shape $\operatorname{srmBm} Z_{g}^{(s)}$ is defined almost surely as the unique fixed point of $\Lambda_{\alpha^{\prime}, \beta^{\prime}}^{(s)}$ :

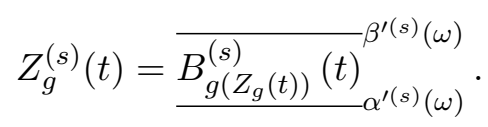

Theorem 3.2.

Almost surely:

$$
\alpha_{Z_{g}^{(s)}}(t, \omega)=g\left(Z_{g}^{(s)}(t, \omega)\right)
$$


The proof is left to the reader.

Remark 3.13. The same results as above hold if $s$ is only assumed to be $b+\epsilon$ Hölder-continuous for an $\epsilon>0$.

The following obvious proposition states that one indeed controls the mean shape of the process at each point. Note that this entails that the pointwise regularity is also controlled at each point through $g$.

Proposition 3.14. Almost surely, for all $t \in[0,1]$ :

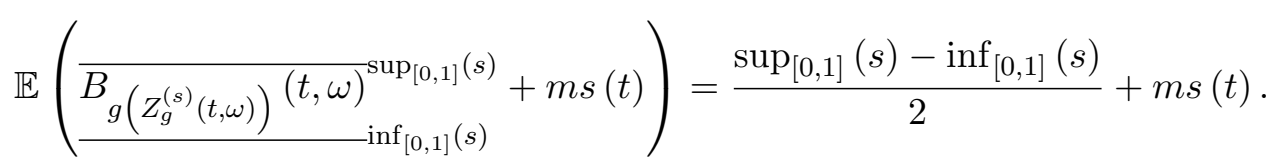

The reader who is consulting an electronic version of this work will find on Figure 5 an animation showing the effect of changing $s$ and $m$ on a prescribed shape srmBm with $g(x)=x$ (click on graph to launch the animation). The value of $m$ is depicted on the left pane. On the right pane, $s$ is drawn as a thick cyan line, while the process is the thin blue line. Finally, the red line is the estimated pointwise exponent at each time, which should be (and approximately is) equal to the process itself. Estimation issues for srmBm will be addressed in a forthcoming article.

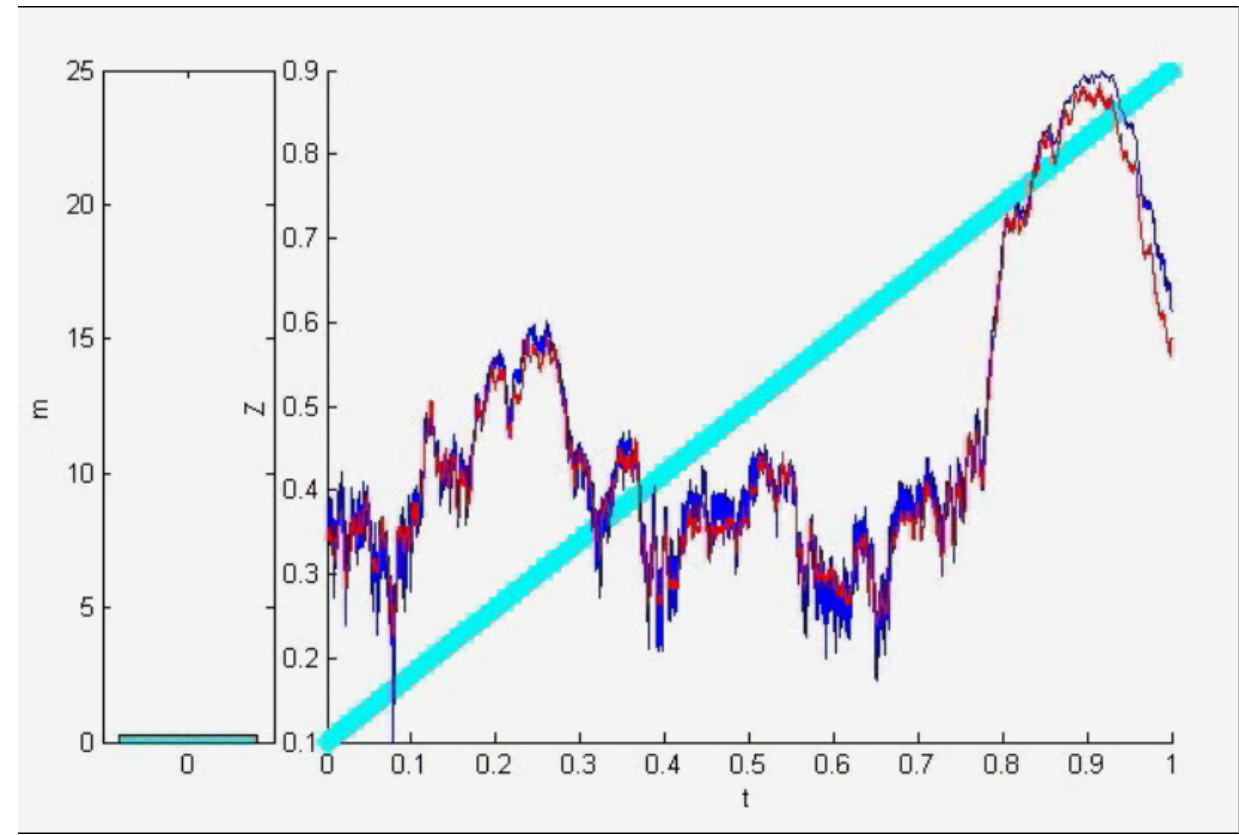

Figure 5: An animation showing the effect of changing the shape $s$ and mixing parameter $m$ of a prescribed shape srmBm with $g(x)=x$.

\section{Self-Regulating Random Midpoint Displace- ment Process}

In this section, we propose a totally different way of building a self-regulating process, which is based on P. Lévy's celebrated construction of Brownian motion 
through random midpoint displacement. The resulting process will not be drawn on a field, which has both some advantages and drawbacks as we will see.

\subsection{Definition and basic properties}

Recall the definition of the "triangle" function:

$$
\varphi(t)=\left\{\begin{array}{lcc}
2 t & \text { for } & t \in[0,1 / 2] \\
1-2 t & \text { for } & t \in[1 / 2,1] \\
0 & \text { otherwise. } &
\end{array}\right.
$$

Define $\varphi_{j k}(t)=\varphi\left(2^{j} t-k\right)$, for $j \in \mathbb{N}, k=0, \ldots, 2^{j}-1$.

Let $Z_{j k}$ be i.i.d. random variables following an $N(0,1)$ law. It is well known that

$$
B=\sum_{j=0}^{\infty} \sum_{k=0}^{2^{j}-1} 2^{-j / 2} \varphi_{j k} Z_{j k}
$$

is a representation of Brownian bridge. We remark that the factor $1 / 2$ in the expression $2^{-j / 2} \varphi_{j k} Z_{j k}$ corresponds to the constant pointwise Hölder exponent of the process. Heuristically, the term $2^{-j / 2} \varphi_{j k}$ entails a variation with amplitude $2^{-j / 2}$ and duration $2^{-j}$. In other words, variations of time length $h=2^{-j}$ are of the order of $h^{1 / 2}$. It is easy to prove, for instance using Theorem 4.1 below, that the modified process $\sum_{j, k} 2^{-j \alpha} \varphi_{j k}(t) Z_{j k}$ has almost surely everywhere Hölder exponent $\alpha$ for $\alpha \in(0,1)$. We shall take advantage of this fact to build in an iterative way a process $X$ verifying almost surely $\alpha_{X}(t)=g(X(t))$ for all $t$, where $g$ is again a $C^{1}$ deterministic function. We shall require that $g$ ranges in $[a, b]$ with $0<a \leq b$ (the condition $[a, b] \subset(0,1)$ is not necessary at this point).

It turns out that the Gaussian character of the random variables $Z_{j k}$ is not crucial for our purpose. Rather, we will need the following assumption:

Assumption $\mathcal{A}$ :

There exists $c \in(0, a)$ such that, almost surely, there exists $N$ in $\mathbb{N}$ with:

$$
\forall j \geq N, \max _{k=0 . .2^{j}-1}\left|Z_{j k}\right| \leq 2^{j c} .
$$

Assumption $\mathcal{A}$ is fulfilled for any $c \in(0, a)$ if the $\left(Z_{j k}\right)_{j, k}$ follow an $N(0,1)$ law. It also verified if they follow for instance an $\alpha$-stable law provided $a$ is large enough.

A self-regulating random midpoint displacement process (srmdp) is defined as follows:

Theorem-Definition 4.1. Let $g$ be a $C^{1}$ function defined on $\mathbb{R}$ and ranging in $[a, b] \subset \mathbb{R}_{+}^{*}$. Let $Z_{j k}$ be i.i.d. centred random variables verifying Assumption $\mathcal{A}$. Set $X_{-1} \equiv 0$ and define the sequence of processes $\left(X_{j}\right)_{j \in \mathbb{N}}$ by:

$$
X_{j}(t)=X_{j-1}(t)+\sum_{k=0}^{2^{j}-1} 2^{-j g\left(X_{j-1}\left(\left(k+\frac{1}{2}\right) 2^{-j}\right)\right)} Z_{j k} \varphi_{j k}(t) .
$$

Almost surely, the sequence $\left(X_{j}\right)_{j \in \mathbb{N}}$ converges uniformly to a continuous process $X$, called srmdp.

Note that the range of $X$ is essentially determined by the one of the random variables $Z_{j k}$. Choosing bounded or unbounded $Z_{j k}$ leads to the same property for $X$. 
Proof. For all $t$ :

$$
X_{j}(t)-X_{j-1}(t)=\sum_{k=0}^{2^{j}-1} 2^{-j g\left(X_{j-1}\left(\left(k+\frac{1}{2}\right) 2^{-j}\right)\right)} Z_{j k} \varphi_{j k}(t)
$$

Since the $\varphi_{j k}$ have disjoint supports:

$$
\left\|X_{j}-X_{j-1}\right\|_{\infty} \leq 2^{-j a} \max _{k=1 \ldots 2^{j-1}}\left|Z_{j k}\right|
$$

Assumption $\mathcal{A}$ entails that $\left(X_{j}\right)_{j \in \mathbb{N}}$ converges almost surely in $C\left([0,1],\|\cdot\|_{\infty}\right)$ to a continuous process $X$.

Proposition 4.1. Assume that $Z_{0,0}$ belongs to $L^{2}(\Omega)$. Then the sequence $\left(X_{j}\right)_{j \in \mathbb{N}}$ converges to $X$ in $L^{2}(\Omega \times[0,1])$.

Proof. The random variables $Z_{j k}$ are independent, and, for all $j, k, Z_{j k}$ is independent of $X_{j-1}(t)$ for all $t$. As a consequence:

$$
\mathbb{E}\left(\left(X_{j-1}(t)-X_{j}(t)\right)^{2}\right)=\sum_{k=0}^{2^{j}-1} \mathbb{E}\left(2^{-2 j g\left(X_{j-1}\left(\left(k+\frac{1}{2}\right) 2^{-j}\right)\right)}\right) \mathbb{E}\left(Z_{j k}^{2}\right) \varphi_{j k}^{2}(t)
$$

Thus:

$$
\begin{aligned}
\int_{0}^{1} \mathbb{E}\left(\left(X_{j-1}(t)-X_{j}(t)\right)^{2}\right) d t & \leq 2^{-2 j a} \mathbb{E}\left(Z_{0,0}^{2}\right) \sum_{k=1}^{2^{j}-1} \int_{0}^{1} \varphi_{j k}^{2}(t) d t \\
& \leq 2^{-2 j a} \mathbb{E}\left(Z_{0,0}^{2}\right) \int_{0}^{1} \varphi^{2}(t) d t
\end{aligned}
$$

which entails convergence in $L^{2}(\Omega \times[0,1])$ to a process $Y$ which has to verify $X=Y$ almost surely.

Remark 4.2. One can show similarly that convergence holds in $L^{p}(\Omega)$ for $p>0$. In fact, $L^{p}(\Omega)$ convergence does not require Assumption $\mathcal{A}$ to be verified, but only that the $\left(Z_{j k}\right)_{j, k}$ be in $L^{p}(\Omega)$, as it may easily be checked.

We observe the following simple facts:

Proposition 4.3. Assume that $Z_{0,0}$ belongs to $L^{p}(\Omega), p \in \mathbb{R}_{+}^{*}$. Then, for all $t \in$ $[0,1]$,

$$
\mathbb{E}\left(\left|X_{n}(t)-X(t)\right|^{p}\right) \rightarrow 0
$$

Proof. Fix $t \in[0,1]$. Then:

$$
X_{j}(t)-X_{j-1}(t)=\sum_{k=0}^{2^{j}-1} 2^{-j g\left(X_{j-1}\left(\left(k+\frac{1}{2}\right) 2^{-j}\right)\right)} Z_{j k} \varphi_{j k}(t) .
$$

At most one term in the sum above is non-zero, and thus

$$
\mathbb{E}\left(\left|X_{j}(t)-X_{j-1}(t)\right|^{p}\right) \leq 2^{-j a p} \mathbb{E}\left(\left|Z_{0,0}\right|^{p}\right) .
$$

This entails that the sequence $\left(X_{j}(t)\right)_{j \in \mathbb{N}}$ converges in $L^{p}(\Omega)$ to a random variable $U$. Since $\left(X_{j}(t)\right)_{j \in \mathbb{N}}$ converges almost surely to $X(t)$ by Theorem-Definition 4.1 , we deduce that $U=X(t)$. 
Corollary 4.4. Assume that $Z_{0,0}$ belongs to $L^{1}(\Omega)$. Then, for all $t$ :

$$
\mathbb{E}(X(t))=0 .
$$

Proof. A straightforward recurrence shows that $\mathbb{E}\left(X_{n}(t)\right)=0$ for all $n$ and all $t$. The results then follows from Proposition 4.3 with $p=1$.

Proposition 4.5. Assume that $Z_{0,0}$ belongs to $L^{2}(\Omega)$. Then, for all $t$ :

$$
\mathbb{E}\left(X(t)^{2}\right)=\mathbb{E}\left(Z_{0,0}^{2}\right) \sum_{j=0}^{\infty} \sum_{k=0}^{2^{j}-1} \mathbb{E}\left(2^{-2 j g\left(X_{j-1}\left(\left(k+\frac{1}{2}\right) 2^{-j}\right)\right)}\right) \varphi_{j k}(t)^{2} .
$$

Proof. Again by independence of the sequence $\left(Z_{j k}\right)_{j, k}$ and independence of $Z_{j k}$ from $X_{j-1}(x)$ for all $x$ :

$$
\mathbb{E}\left(X_{j}(t)^{2}\right)=\mathbb{E}\left(X_{j-1}(t)^{2}\right)+\mathbb{E}\left(Z_{0,0}^{2}\right) \sum_{k=0}^{2^{j}-1} \mathbb{E}\left(2^{-2 j g\left(X_{j-1}\left(\left(k+\frac{1}{2}\right) 2^{-j}\right)\right)}\right) \varphi_{j k}(t)^{2} .
$$

A straightforward recurrence yields that, for all $j \geq 0$ :

$$
\mathbb{E}\left(X_{j}(t)^{2}\right)=\mathbb{E}\left(Z_{0,0}^{2}\right) \sum_{l=0}^{j} \sum_{k=0}^{2^{l}-1} \mathbb{E}\left(2^{-2 l g\left(X_{l-1}\left(\left(k+\frac{1}{2}\right) 2^{-l}\right)\right)}\right) \varphi_{l k}(t)^{2} .
$$

and the result follows by letting $j$ tend to infinity and using Proposition 4.3 (the sequence converges since, for all $l$, at most one term in the sum is non-zero).

One deduces the obvious bounds:

$$
\mathbb{E}\left(Z_{0,0}^{2}\right) \sum_{j=0}^{\infty} 2^{-2 j b} \sum_{k=0}^{2^{j}-1} \varphi_{j k}(t)^{2} \leq \mathbb{E}\left(X(t)^{2}\right) \leq \mathbb{E}\left(Z_{0,0}^{2}\right) \sum_{j=0}^{\infty} 2^{-2 j a} \sum_{k=0}^{2^{j}-1} \varphi_{j k}(t)^{2},
$$

and, by noting that at most one $\varphi_{j k}(t)$ is non-zero for all $j$ :

$$
\mathbb{E}\left(X(t)^{2}\right) \leq \frac{\mathbb{E}\left(Z_{0,0}^{2}\right)}{1-2^{-2 a}}
$$

\subsection{Self-regulation}

Our main result in this section is the following, which describes the local and pointwise Hölder regularity of $X$.

Theorem 4.1. 1. Assume that $g$ ranges in $[a, b] \subset(0,1)$ and that the random variables $\left(Z_{j k}\right)_{j, k}$ verify Assumption $\mathcal{A}$. Then, almost surely, for all t:

$$
\alpha_{X}^{l}(t) \geq g(X(t))-c .
$$

2. If the random variables $\left(Z_{j k}\right)_{j, k}$ are such that, for all $x \geq 0$ :

$$
\mathbb{P}\left(\left|Z_{0,0}\right| \leq x\right) \leq x^{\gamma}
$$

for some $\gamma>0$, then, almost surely, for all $t$ :

$$
\alpha_{X}(t) \leq g(X(t)) .
$$


Remark 4.6. Theorem 4.1 extends to arbitrary intervals $[a, b] \subset(0, \infty)$ provided one uses sufficiently smooth wavelets in place of the triangle function in the definition of $X$.

Proof of Theorem 4.1.

See Appendix 6.3.

Theorem 4.1 entails that, in the Gaussian case, an srmdp is indeed self-regulating:

Corollary 4.7. Assume that the random variables $\left(Z_{j k}\right)_{j, k}$ are Gaussian and that $g$ ranges in $[a, b] \subset(0,1)$. Then, almost surely, for all $t$ :

$$
\alpha_{X}^{l}(t)=\alpha_{X}(t)=g(X(t)) .
$$

In addition, if $g$ is not constant, then $X$ is not a Gaussian process.

Proof of Corollary 4.7.

This a simple consequence of the facts that one always has $\alpha_{X}^{l}(t) \leq \alpha_{X}(t)$ and that Assumption $\mathcal{A}$ is verified for all $c>0$ in the Gaussian case.

Remark 4.8. From the definition, it is easy to see that, at any dyadic point, the value of $X$ is determined in a finite number of steps. This remark plus the fact that, for Gaussian $\left(Z_{j k}\right)_{j, k}, X_{j}$ is Gaussian conditionally on the filtration generated that $X_{j-1}$ (although $X$ is non-Gaussian for non-constant $g$ ) are instrumental for solving the estimation problem, that is, inferring the function $g$ from sampled data [19].

In addition, the same remark implies that $X$ may be simulated in an exact way through Formula (21) provided the number of samples $N$ is a power of 2. When this is not the case, one may always choose the smallest integer $n$ such that $N<2^{n}$, generate $X$ on $2^{n}$ points, and keep only the $N$ first samples. While this procedure may at most double the number of computations, this is not a serious drawback in practice as the computational cost of the synthesis procedure is linear in N. See Figure 6 for simulated realizations of srmdps.

Figure 6 displays three realisations of $X$ for an increasing $g$, obtained with FracLab.
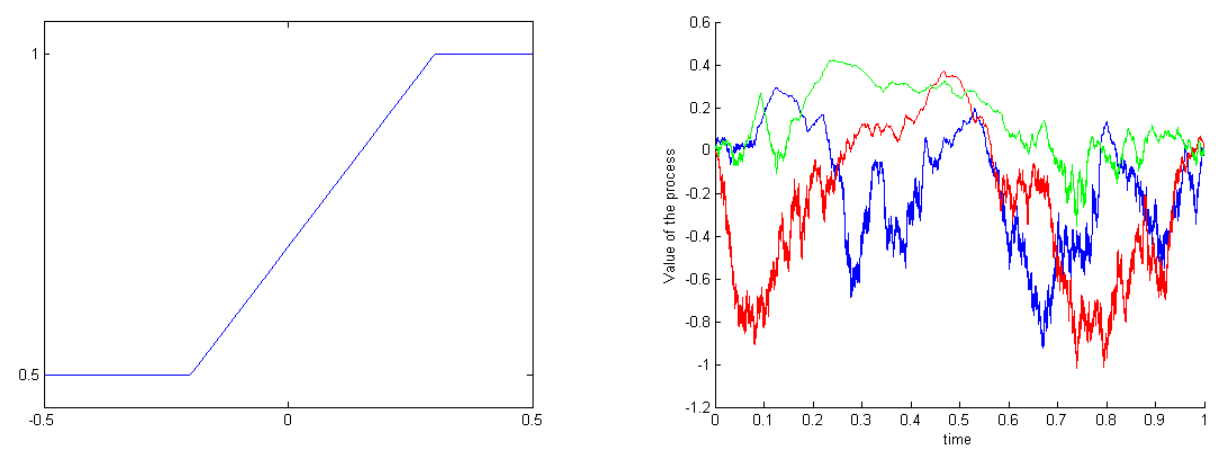

Figure 6: Left: self-regulating function $g$. Right: three realisations of $X$. One verifies that the process is more regular at points where it has larger amplitude. 


\section{Future work}

We have presented two different constructions of self-regulating processes in Sections 3 and 4 . One may wonder how they compare. On the one hand, self-regulating midpoint displacement processes are certainly easier to work with, at least in the Gaussian case, as they have a "tree structure" that makes them conditionally Gaussian. As a consequence, simulating them is simpler, and it is not too difficult to construct an estimator for the self-regulating function $g$ [19]. On the other hand, we believe they have a less rich structure than self-regulating multifractional Brownian motions. More precisely, when one takes $g=H=$ constant, one gets simply an fBm with exponent $H$ in the srmBm case. In the srmdp case, one obtains a process, say $X$, whose pointwise Hölder exponent is also everywhere equal to $H$ almost surely. However, while the increments of $\mathrm{fBm}$ display long range dependence for $H>1 / 2$, this is not the case for $X$ (this is an easy computation left to the reader). We conjecture that the same difference holds for the self-regulating versions, although proving this fact seems out of reach at this time. Indeed, even the marginal laws of both processes are not known, let alone their covariance structure. Nonetheless, such a conjecture is supported by visual inspection of realizations of the processes, as well as by numerical experiments.

Another interesting study would be to compute the multifractal spectra of our processes. Indeed, since their Hölder functions are random, the question arises whether their multifractal spectra share the same property (recall that, for instance, the Hölder function of a Lévy process is random -except in the Brownian case-, while its multifractal spectrum is deterministic). We conjecture that this is the case, as the range of the exponents is itself random. Again, this does not appear to be an easy problem, as it seems to require characterizing the local time of the processes.

Finally, it is clear that much more general "self-regulating relations" than the one we have considered here could be studied. They could for instance involve a second independent or correlated process, primitives or derivatives of $X$ and/or of $\alpha_{X}$, or other local features, such as the 2 -microlocal spectrum or the stability index in the case of multistable processes. Accounting for a random coupling $g$ would also be interesting. Two of these generalizations were alluded to in Section 3.2. More general self-regulating relations would in particular be useful in view of applications outside of mathematics (e.g. in financial modelling).

\section{Appendix}

\subsection{Proof of Theorem 2.1}

For simplicity, we will restrict to the case where $g$ ranges in $[\alpha, \beta]$ with $\beta<1$.

The proof of the inequality $\alpha_{h^{*}}(t) \leq g\left(h^{*}(t)\right)$ is exactly the same as the one in $[11$, Proposition 7].

For the reverse inequality, we note first that the proof of Proposition 7 in [11] cannot not possibly apply, since it requires that $\alpha_{h}(t)>g(h(t))$, which of course does not hold here. Nevertheless, it is easy to adapt it in our frame. 
Fix $t$ and $\varepsilon$. Then:

$$
\begin{aligned}
h^{*}(t+\varepsilon)-h^{*}(t) & =W_{g\left(h^{*}\right)}(t+\varepsilon)-W_{g\left(h^{*}\right)}(t) \\
& =\sum_{n=1}^{\infty}\left(\lambda^{-n g\left(h^{*}(t+\varepsilon)\right)} \sin \left(\lambda^{n}(t+\varepsilon)\right)-\lambda^{-n g\left(h^{*}(t)\right)} \sin \left(\lambda^{n} t\right)\right) \\
& =A+A^{\prime},
\end{aligned}
$$

with :

$$
\begin{aligned}
A & =\sum_{n=1}^{\infty}\left(\lambda^{-n g\left(h^{*}(t+\varepsilon)\right)}-\lambda^{-n g\left(h^{*}(t)\right)}\right) \sin \left(\lambda^{n}(t+\varepsilon)\right), \\
A^{\prime} & =\sum_{n=1}^{\infty} \lambda^{-n g\left(h^{*}(t)\right)}\left(\sin \left(\lambda^{n}(t+\varepsilon)\right)-\sin \left(\lambda^{n} t\right)\right) .
\end{aligned}
$$

Reasoning as above, one gets, for $|A|$ :

$$
\begin{aligned}
|A| & \leq \sum_{n=1}^{\infty}\left|\lambda^{-n g\left(h^{*}(t+\varepsilon)\right)}-\lambda^{-n g\left(h^{*}(t)\right)}\right| \\
& \leq \ln (\lambda)\left|g\left(h^{*}(t+\varepsilon)\right)-g\left(h^{*}(t)\right)\right| \sum_{n=1}^{\infty} n \lambda^{-n \alpha} \\
& \leq k_{g} \ln (\lambda) \frac{\lambda^{\alpha}}{\left(\lambda^{\alpha}-1\right)^{2}}\left|h^{*}(t+\varepsilon)-h^{*}(t)\right| \\
& =k_{\Phi}\left|h^{*}(t+\varepsilon)-h^{*}(t)\right| .
\end{aligned}
$$

In order to obtain an estimate for $\left|A^{\prime}\right|$, consider the integer $N$ such that:

$$
\lambda^{-(N+1)}<|\varepsilon| \leq \lambda^{-N} .
$$

The finite increments theorem yields

$$
\left|A^{\prime}\right| \leq|\varepsilon| X+2 Y
$$

where:

$$
X=\sum_{n=1}^{N} \lambda^{-n\left(g\left(h^{*}(t)\right)-1\right)} \leq \frac{1}{1-\lambda^{-n\left(g\left(h^{*}(t)\right)-1\right)}}|\varepsilon|^{g\left(h^{*}(t)\right)-1}
$$

and

$$
Y=\sum_{n=N+1}^{\infty} \lambda^{-n g\left(h^{*}(t)\right)} \leq \frac{1}{1-\lambda^{-n g\left(h^{*}(t)\right)}}|\varepsilon|^{g\left(h^{*}(t)\right)} .
$$

As a consequence, there exists a constant $c$ such that:

$$
\left|A^{\prime}\right| \leq c|\varepsilon|^{g\left(h^{*}(t)\right)} .
$$

Going back to 23), one gets:

$$
\begin{aligned}
\left|h^{*}(t+\varepsilon)-h^{*}(t)\right| & \leq|A|+\left|A^{\prime}\right| \\
& \leq k_{\Phi}\left|h^{*}(t+\varepsilon)-h^{*}(t)\right|+c|\varepsilon|^{g\left(h^{*}(t)\right)},
\end{aligned}
$$

or

$$
\left|h^{*}(t+\varepsilon)-h^{*}(t)\right|\left(1-k_{\Phi}\right) \leq c|\varepsilon|^{g\left(h^{*}(t)\right)} .
$$

Finally, since $1-k_{\Phi}>0$ :

$$
\left|h^{*}(t+\varepsilon)-h^{*}(t)\right| \leq \frac{c}{1-k_{\Phi}}|\varepsilon|^{g\left(h^{*}(t)\right)} .
$$

This entails that $\alpha_{h^{*}}(t) \geq g\left(h^{*}(t)\right)$. 


\subsection{Proof of Theorem 3.1}

Theorem 3.1 in [8] states that, for all $t_{0} \in[0,1], \alpha_{Z}\left(t_{0}, \omega\right)=S\left(t_{0}, \omega\right)$ almost surely for an MPRE under condition (11). If $Z_{g}$ is indeed self-regulating, this condition cannot hold, and we need to modify some of the arguments of [8].

The proof of Theorem 3.1 in [8] relies on Lemmas 3.1 to 3.4 of the same article. We explain how to adapt them. First, recall that in [8] the field $B$ is split into a "low frequency " part and a "high frequency" one. More precisely, one first decomposes $B$ on the Lemarié-Meyer wavelet basis $\left\{\psi_{j, k}\right\}_{(j, k) \in \mathbb{Z}^{2}}$ : for all $(t, H)$ in $[0,1] \times[a, b]$,

$$
B_{H}(t)=\sum_{j \in \mathbb{Z}} \sum_{k \in \mathbb{Z}} a_{j, k}(t, H) \epsilon_{j, k}
$$

where $\left(\epsilon_{j, k}\right)_{j, k}$ are independent and identically distributed (i.i.d.) random variables following an $N(0,1)$ law (that is, a centred Gaussian law with variance one) and where the coefficients $a_{j, k}(t, H)$ read:

$$
a_{j, k}(t, H)=\int_{\mathbb{R}} \frac{e^{i t \xi}-1}{i \xi|\xi|^{H-1 / 2}} \hat{\psi}_{j, k}(\xi) d \xi
$$

$\left(\hat{\psi}_{j, k}\right.$ denotes the Fourier transform of $\left.\psi_{j, k}\right)$.

Define for $(x, H) \in \mathbb{R} \times[a, b]$ the function :

$$
\Psi(x, H)=\int_{R} e^{i x \xi} \frac{\hat{\psi}(\xi)}{i \xi|\xi|^{H-1 / 2}} d \xi .
$$

Set $\eta=2^{-j} \xi$. Then:

$$
a_{j, k}(t, H)=2^{-j H}\left(\Psi\left(2^{j} t-k, H\right)-\Psi(-k, H)\right) .
$$

The frequency decomposition of $B$ reads:

$$
B_{H}(t)=\dot{B}_{H}(t)+\ddot{B}_{H}(t)
$$

where:

$$
\begin{aligned}
& \dot{B}_{H}(t)=\sum_{j=-\infty}^{-1} \sum_{k \in \mathbb{Z}} a_{j, k}(t, H) \varepsilon_{j, k}=\sum_{j=1}^{\infty} \sum_{k \in \mathbb{Z}} 2^{j H} \varepsilon_{-j, k}\left(\Psi\left(2^{-j} t-k, H\right)-\Psi(-k, H)\right), \\
& \ddot{B}_{H}(t)=\sum_{j=0}^{\infty} \sum_{k \in \mathbb{Z}} a_{j, k}(t, H) \varepsilon_{j, k}=\sum_{j=0}^{\infty} \sum_{k \in \mathbb{Z}} 2^{-j H} \varepsilon_{j, k}\left(\Psi\left(2^{j} t-k, H\right)-\Psi(-k, H)\right) .
\end{aligned}
$$

Proposition 6.1. [8]

The field $\left\{\dot{B}_{H}(t)\right\}_{(t, H) \in[0,1] \times[a, b]}$ is almost surely $C^{\infty}$ on $[0,1] \times[a, b]$.

It is proved in [8] that Proposition 3.1 holds for both $\ddot{B}_{H}$ and $B_{H}$. By replacing the random variable $C_{2}(\omega)$ appearing in $(13)$ by:

$$
\max \left(\sup _{(H, t) \in[a, b] \times[0,1]}\left|\left(\frac{\partial}{\partial H}\right) B(H, t, \omega)\right|, \sup _{(H, t) \in[a, b] \times[0,1]}\left|\left(\frac{\partial}{\partial H}\right) \ddot{B}(H, t, \omega)\right|\right),
$$


we may and will assume that 13 holds simultaneously for $\ddot{B}$ and $B$ with the same $C_{2}(\omega)$ (this will modify Condition $\mathcal{C}$ accordingly).

Lemma 3.2 of [8] states that, for $t_{0}$ fixed in $[0,1]$ and any stochastic process $S$ valued in $[a, b]$, the series

$$
\ddot{T}_{t_{0}}(t, \omega)=\sum_{j=0}^{\infty} \sum_{k \in \mathbb{Z}} 2^{-j S\left(t_{0}, \omega\right)} \varepsilon_{j, k}(\omega) \Psi\left(2^{j} t-k, S\left(t_{0}, \omega\right)\right) .
$$

is almost surely uniformly convergent in $t$ on any compact subset of $\mathbb{R}$. Moreover, there exists an almost surely finite random variable $C_{5}>0$ such that, for all $t$ in $\mathbb{R}$ :

$$
\left|\ddot{T}_{t_{0}}(t, \omega)\right| \leq C_{5}(\omega) \sqrt{\log (2+|t|)} .
$$

This proof of this lemma does not rely on assumption (11) and we shall apply it with $S=g\left(Z_{g}\right)$ which indeed ranges in $[a, b]$. Thus, in our case, $\ddot{T}_{t_{0}}$ is defined as:

$$
\ddot{T}_{t_{0}}(t, \omega)=\sum_{j=0}^{\infty} \sum_{k \in \mathbb{Z}} 2^{-j g\left(Z_{g}\left(t_{0}, \omega\right)\right)} \varepsilon_{j, k}(\omega) \Psi\left(2^{j} t-k, g\left(Z_{g}\left(t_{0}, \omega\right)\right)\right) .
$$

Likewise, Lemma 3.4 of [8], which deals with various properties of the function $\tilde{\Psi}$ :

$$
\tilde{\Psi}(x, H)=i \int_{R} e^{i x \xi} \xi|\xi|^{H-1 / 2} \hat{\psi}(\xi) d \xi
$$

still holds true in our frame, since it is of purely analytical nature.

In contrast, Lemmas 3.1 et 3.3 of [8] use crucially condition (11), and we now prove a version of them adapted to our case.

Lemma 6.2. Counterpart of Lemma 3.1 of [8]

Let $J$ be an interval in $[0,1]$. Almost surely, for all $\epsilon>0$ and all $\left(t^{\prime}, t^{\prime \prime}\right) \in J^{2}$ :

$$
\left|Z_{g}\left(t^{\prime}, \omega\right)-Z_{g}\left(t^{\prime \prime}, \omega\right)\right| \leq C(\omega)\left|t^{\prime}-t^{\prime \prime}\right|^{m(\omega)-\epsilon}
$$

where $C(\omega)$ is an almost surely finite random variable and $m(\omega)=\inf _{t \in J} g\left(Z_{g}(t, \omega)\right)$.

Proof.

By definition of $Z_{g}$ :

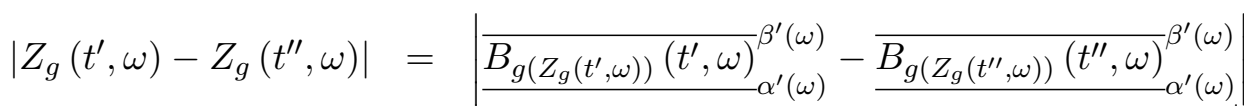

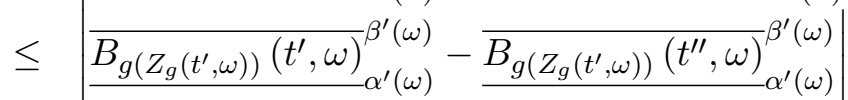

$$
\begin{aligned}
& +\left|\frac{{\overline{B g\left(Z_{g}\left(t^{\prime}, \omega\right)\right)}\left(t^{\prime \prime}, \omega\right)}_{\alpha^{\prime}(\omega)}^{\beta^{\prime}(\omega)}-{\overline{B_{g\left(Z_{g}\left(t^{\prime \prime}, \omega\right)\right)}\left(t^{\prime \prime}, \omega\right)}}_{\alpha^{\prime}(\omega)}{ }^{\prime}(\omega)}{}\right| .
\end{aligned}
$$

Using (12), 13) and then the Lipschitz property of $g$, one gets (we do not indicate the dependency in $\omega$ for better readability):

$$
\begin{aligned}
\left|Z_{g}\left(t^{\prime}\right)-Z_{g}\left(t^{\prime \prime}\right)\right| & \leq \frac{\beta^{\prime}-\alpha^{\prime}}{M_{B}-m_{B}}\left(C_{1}\left|t^{\prime}-t^{\prime \prime}\right|^{m-\varepsilon}+C_{2}\left|g\left(Z_{g}\left(t^{\prime}\right)\right)-g\left(Z_{g}\left(t^{\prime \prime}\right)\right)\right|\right) \\
& \leq \frac{\beta^{\prime}-\alpha^{\prime}}{M_{B}-m_{B}}\left(C_{1}\left|t^{\prime}-t^{\prime \prime}\right|^{m-\varepsilon}+C_{2} k_{g}\left|Z_{g}\left(t^{\prime}\right)-Z_{g}\left(t^{\prime}\right)\right|\right),
\end{aligned}
$$


or:

$$
\left|Z_{g}\left(t^{\prime}\right)-Z_{g}\left(t^{\prime \prime}\right)\right|\left(1-\frac{\left(\beta^{\prime}-\alpha^{\prime}\right) C_{2} k_{g}}{M_{B}-m_{B}}\right) \leq \frac{\beta^{\prime}-\alpha^{\prime}}{M_{B}-m_{B}} C_{1}\left|t^{\prime}-t^{\prime \prime}\right|^{m-\varepsilon} .
$$

Condition $\mathcal{C}$ says that:

$$
\frac{\left(\beta^{\prime}(\omega)-\alpha^{\prime}(\omega)\right) C_{2}(\omega) k_{g}}{M_{B}(\omega)-m_{B}(\omega)}<1
$$

and the results follows.

Lemma 6.3. Counterpart of Lemma 3.3 in [8]

Fix $t_{0}$ in $[0,1]$. For all $\omega$ in $\Omega_{1}^{*}$ :

$$
\alpha_{Z_{g}}\left(t_{0}, \omega\right)=g\left(Z_{g}\left(t_{0}, \omega\right)\right) \Leftrightarrow \alpha_{\ddot{T}_{t_{0}}}\left(t_{0}, \omega\right)=g\left(Z_{g}\left(t_{0}, \omega\right)\right) .
$$

Proof.

Define:

$$
\begin{aligned}
\Delta_{h} Z_{g}\left(t_{0}, \omega\right) & :=Z_{g}\left(t_{0}+h, \omega\right)-Z_{g}\left(t_{0}, \omega\right) \\
& =\frac{\beta^{\prime}(\omega)-\alpha^{\prime}(\omega)}{M_{B}(\omega)-m_{B}(\omega)}\left(B_{g\left(Z_{g}\left(t_{0}+h, \omega\right)\right)}\left(t_{0}+h, \omega\right)-t B_{g\left(Z_{g}\left(t_{0}, \omega,\right)\right)}\left(t_{0}, \omega\right)\right)
\end{aligned}
$$

and

$$
\begin{aligned}
\Delta_{h} \ddot{T}_{t_{0}}\left(t_{0}, \omega\right) & :=\ddot{T}_{t_{0}}\left(t_{0}+h, \omega\right)-\ddot{T}_{t_{0}}\left(t_{0}, \omega\right) \\
& =\ddot{B}_{g\left(Z_{g}\left(t_{0}, \omega\right)\right)}\left(t_{0}+h, \omega\right)-\ddot{B}_{g\left(Z_{g}\left(t_{0}, \omega\right)\right)}\left(t_{0}, \omega\right),
\end{aligned}
$$

which follows from $(32)$ and 30 .

One computes (we omit $\omega$ for better readability):

$$
\left|\Delta_{h} Z_{g}\left(t_{0}\right)\right|=\frac{\beta^{\prime}-\alpha^{\prime}}{M_{B}-m_{B}}\left|B_{g\left(Z_{g}\left(t_{0}+h\right)\right)}\left(t_{0}+h\right)-B_{g\left(Z_{g}\left(t_{0}\right)\right)}\left(t_{0}\right)\right|
$$

and

$$
\begin{aligned}
& \left|B_{g\left(Z_{g}\left(t_{0}+h\right)\right)}\left(t_{0}+h\right)-B_{g\left(Z_{g}\left(t_{0}\right)\right)}\left(t_{0}\right)\right| \leq\left|B_{g\left(Z_{g}\left(t_{0}+h\right)\right)}\left(t_{0}+h\right)-B_{g\left(Z_{g}\left(t_{0}\right)\right)}\left(t_{0}+h\right)\right| \\
& +\left|B_{g\left(Z_{g}\left(t_{0}\right)\right)}\left(t_{0}+h\right)-B_{g\left(Z_{g}\left(t_{0}\right)\right)}\left(t_{0}\right)\right| \\
& \leq C_{2} \mid g\left(Z_{g}\left(t_{0}+h\right)-g\left(Z_{g}\left(t_{0}\right) \mid\right.\right. \\
& +\left|\dot{B}_{g\left(Z_{g}\left(t_{0}\right)\right)}\left(t_{0}+h\right)-\dot{B}_{g\left(Z_{g}\left(t_{0}\right)\right)}\left(t_{0}\right)\right| \\
& +\left|\ddot{B}_{g\left(Z_{g}\left(t_{0}\right)\right)}\left(t_{0}+h\right)-\ddot{B}_{g\left(Z_{g}\left(t_{0}\right)\right)}\left(t_{0}\right)\right| \\
& \leq C_{2} k_{g}\left|\Delta_{h} Z_{g}\left(t_{0}\right)\right|+C_{3}|h|+\left|\Delta_{h} \ddot{T}_{t_{0}}\left(t_{0}\right)\right|
\end{aligned}
$$

where we have used Proposition 6.1 which entails that there exists an almost surely finite random variable $C_{3}(\omega)$ such that $\left|\dot{B}_{g\left(Z_{g}\left(t_{0}\right)\right)}\left(t_{0}+h\right)-\dot{B}_{g\left(Z_{g}\left(t_{0}\right)\right)}\left(t_{0}\right)\right| \leq C_{3}|h|$. 
Coming back to $\Delta_{h} Z_{g}$, one gets:

$$
\left|\Delta_{h} Z_{g}\left(t_{0}\right)\right|\left(1-C_{2} k_{g} \frac{\beta^{\prime}-\alpha^{\prime}}{M_{B}-m_{B}}\right) \leq C_{3} \frac{\beta^{\prime}-\alpha^{\prime}}{M_{B}-m_{B}}|h|+\frac{\beta^{\prime}-\alpha^{\prime}}{M_{B}-m_{B}}\left|\Delta_{h} \ddot{T}_{t_{0}}\left(t_{0}\right)\right| \text {. }
$$

One also has the lower bound:

$$
\begin{aligned}
& \left|B_{g\left(Z_{g}\left(t_{0}+h\right)\right)}\left(t_{0}+h\right)-B_{g\left(Z_{g}\left(t_{0}\right)\right)}\left(t_{0}\right)\right| \geq-\left|B_{g\left(Z_{g}\left(t_{0}+h\right)\right)}\left(t_{0}+h\right)-B_{g\left(Z_{g}\left(t_{0}\right)\right)}\left(t_{0}+h\right)\right| \\
& -\left|\dot{B}_{g\left(Z_{g}\left(t_{0}\right)\right)}\left(t_{0}+h\right)-\dot{B}_{g\left(Z_{g}\left(t_{0}\right)\right)}\left(t_{0}\right)\right| \\
& +\left|\ddot{B}_{g\left(Z_{g}\left(t_{0}\right)\right)}\left(t_{0}+h\right)-\ddot{B}_{g\left(Z_{g}\left(t_{0}\right)\right)}\left(t_{0}\right)\right| \\
& \geq \quad-C_{2} k_{g}\left|\Delta_{h} Z_{g}\left(t_{0}\right)\right|-C_{3}|h|+\left|\Delta_{h} \ddot{T}_{t_{0}}\left(t_{0}\right)\right| \text {. }
\end{aligned}
$$

(38) and (39) yield:

$$
\left|\Delta_{h} Z_{g}\left(t_{0}\right)\right|\left(1-C_{2} k_{g} \frac{\beta^{\prime}-\alpha^{\prime}}{M_{B}-m_{B}}\right)-C_{3} \frac{\beta^{\prime}-\alpha^{\prime}}{M_{B}-m_{B}}|h| \leq \frac{\beta^{\prime}-\alpha^{\prime}}{M_{B}-m_{B}}\left|\Delta_{h} \ddot{T}_{t_{0}}\left(t_{0}\right)\right|
$$

and

$$
\frac{\beta^{\prime}-\alpha^{\prime}}{M_{B}-m_{B}}\left|\Delta_{h} \ddot{T}_{t_{0}}\left(t_{0}\right)\right| \leq\left|\Delta_{h} Z_{g}\left(t_{0}\right)\right|\left(1+C_{2} k_{g} \frac{\beta^{\prime}-\alpha^{\prime}}{M_{B}-m_{B}}\right)+C_{3} \frac{\beta^{\prime}-\alpha^{\prime}}{M_{B}-m_{B}}|h| .
$$

Noting that $|h|^{1-g\left(Z_{g}\left(t_{0}, \omega\right)\right)}$ tends to 0 when $h$ tends to 0 since $g$ is everywhere strictly smaller than 1 yields the announced result.

Remark 6.4. The proof of the lemma shows in fact that:

$$
\alpha_{Z_{g}}\left(t_{0}, \omega\right)=r<1 \Leftrightarrow \alpha_{\ddot{T}_{t_{0}}}\left(t_{0}, \omega\right)=r<1 .
$$

The rest of the proof of our Theorem 3.1 goes exactly along the same lines as the one of Theorem 3.1 in [8].

\subsection{Proof of Theorem 4.1}

Let us first show that $\alpha_{X}^{l}(t) \geq g(X(t))-c$ almost surely for all $t$. Choose $\delta>0$ such that $c+\delta<a$. By uniform continuity of $g \circ X$, there exists $\epsilon>0$ such that, for all $x, t$ in $[0,1]$ with $|x-t|<\epsilon$ :

$$
g(X(x)) \geq g(X(t))-\delta .
$$

For an $\omega$ such that Assumption $\mathcal{A}$ is fulfilled, choose $N \in \mathbb{N}$ such that for all $j \geq N$ :

$$
\max _{k=1 \ldots 2^{j-1}}\left|Z_{j k}\right| \leq 2^{j c} .
$$

Increasing $N$ if necessary, one may also guarantee that:

$$
\sum_{N}^{\infty} j .2^{-j(a-c)} \leq \frac{1}{4 \ln 2 \cdot\left\|g^{\prime}\right\|_{\infty}} .
$$

For any $x, t$ in $[0,1]$ such that $|x-t|<\epsilon$, let $j_{0}$ denote the integer verifying $2^{-j_{0}}<|x-t| \leq 2^{-j_{0}+1}$. In addition, for any integer $j$, let: 
- $t_{j}$ be the integer such that $t \in\left[t_{j} 2^{-j},\left(t_{j}+1\right) 2^{-j}\right)$. In other words, $t_{j}$ is the integer such that $t$ is in the support of $\varphi_{j, t_{j}}$.

- $x_{j}$ be the integer such that $x \in\left[x_{j} 2^{-j},\left(x_{j}+1\right) 2^{-j}\right)$, so that $x$ is in the support of $\varphi_{j, x_{j}}$.

By definition of $X$,

$X(t)-X(x)=X_{N-1}(x)-X_{N-1}(t)+\sum_{j=N}^{\infty} \sum_{k=1}^{2^{j}-1} Z_{j, k}\left(\varphi_{j k}(x) 2^{-j g(X(x))}-\varphi_{j k}(t) 2^{-j g(X(t))}\right)$.

For any fixed $j$, the only non-vanishing terms in the sum over $k$ are the ones with indices $t_{j}$ or $x_{j}$. As a consequence,

$$
|X(t)-X(x)| \leq\left|X_{N-1}(t)-X_{N-1}(x)\right|+\sum_{j=N}^{\infty}\left|Z_{j, t_{j}} A_{j}\right|+\sum_{j=N}^{\infty}\left|Z_{j, x_{j}} B_{j}\right|
$$

where:

$$
\begin{aligned}
& A_{j}=\varphi_{j t_{j}}(t) \cdot 2^{-j \cdot g(X(t))}-\varphi_{j t_{j}}(x) \cdot 2^{-j g(X(x))}, \\
& B_{j}=\varphi_{j x_{j}}(t) \cdot 2^{-j \cdot g(X(t))}-\varphi_{j x_{j}}(x) \cdot 2^{-j g(X(x))} .
\end{aligned}
$$

(note that inequality 43 still holds when $t_{j}=x_{j}$ for some indices $j$ ). Assume for now that $N \leq j_{0}-1$. For $j \in\left[N, \ldots j_{0}-1\right]$, let:

$$
A_{j}=C_{j}+D_{j}
$$

where

$$
C_{j}=\left(\varphi_{j, t_{j}}(t)-\varphi_{j, t_{j}}(x)\right) 2^{-j g(X(t))}
$$

and

$$
D_{j}=\varphi_{j, t_{j}}(x)\left(2^{-j g(X(t))}-2^{-j g(X(x))}\right) .
$$

We seek an upper bound on $\sum_{j=N}^{j_{0}-1}\left|Z_{j, t_{j}} A_{j}\right|$. The finite increments theorem yields:

$$
\left|C_{j}\right| \leq 2^{j}|x-t| 2^{-j g(X(t))},
$$

and, using 41):

$$
\left|Z_{j, t_{j}} C_{j}\right| \leq|x-t| 2^{j(1-g(X(t)+c))}
$$

As a consequence:

$$
\begin{aligned}
\sum_{j=N}^{j_{0}-1}\left|Z_{j, t_{j}} C_{j}\right| & \leq|x-t| \sum_{j=N}^{j_{0}-1} 2^{j(1-g(X(t))+c)} \\
& \leq 2^{-j_{0}+1} \frac{2^{j_{0}(1-g(X(t))+c)}}{2^{1-g(X(t))+c}-1} \\
& \leq 2^{j_{0}(-g(X(t))+c)} \frac{2}{2^{1-b+c}-1} \\
& \leq K_{1}|x-t|^{g(X(t))-c}
\end{aligned}
$$

where $K_{1}=\frac{2}{2^{1-b+c}-1}$.

Let us now estimate $\left|D_{j}\right|$. Clearly,

$$
\left|D_{j}\right| \leq\left|2^{-j g(X(t))}-2^{-j g(X(x))}\right| .
$$


The finite increments theorem yields:

$$
\left|D_{j}\right| \leq|X(x)-X(t)|\left\|g^{\prime}\right\|_{\infty} \cdot \ln 2 . j 2^{-j a} .
$$

Then, with 41):

$$
\sum_{j=N}^{j_{0}-1}\left|Z_{j, t_{j}} D_{j}\right| \leq|X(x)-X(t)|\left\|g^{\prime}\right\|_{\infty} \cdot \ln 2 . \sum_{j=N}^{j_{0}-1} j 2^{-j(a-c)},
$$

and, using 42 :

$$
\sum_{j=N}^{j_{0}-1}\left|Z_{j, t_{j}} D_{j}\right| \leq \frac{1}{4}|X(x)-X(t)| .
$$

Summing up, 44) and 45 yield:

$$
\sum_{j=N}^{j_{0}-1}\left|Z_{j, t_{j}} A_{j}\right| \leq K_{1}|x-t|^{g(X(t))-c}+\frac{1}{4}|X(x)-X(t)| .
$$

Note that the above inequality remains true when $j_{0} \leq N$ with the convention that a sum on an empty set is 0 .

For $j \geq j_{0}, \varphi_{j, t_{j}}(x)=0$, and thus $A_{j}=\varphi_{j t_{j}}(t) .2^{-j \cdot g(X(t))}$. As a consequence:

$$
\begin{aligned}
\sum_{j=j_{0}}^{\infty}\left|Z_{j k} A_{j}\right| & \leq \sum_{j=j_{0}}^{\infty} 2^{j c} 2^{-j g(X(t))} \\
& =\frac{2^{-j_{0}(g(X(t))-c)}}{1-2^{-g(X(t))+c}} \\
& \leq K_{2}|x-t|^{g(X(t))-c}
\end{aligned}
$$

where $K_{2}=\frac{1}{1-2^{-a+c}}$.

Inequalities 46 and 47 yield:

$$
\sum_{j=N}^{\infty}\left|Z_{j k} A_{j}\right| \leq\left(K_{1}+K_{2}\right)|x-t|^{g(X(t))-c}+\frac{1}{4}|X(x)-X(t)| .
$$

Exactly the same estimates as above hold with $x_{j}$ in place of $t_{j}$, and thus:

$$
\begin{aligned}
\sum_{j=N}^{\infty}\left|Z_{j k} B_{j}\right| & \leq\left(K_{1}+K_{2}\right)|x-t|^{g(X(x))-c}+\frac{1}{4}|X(x)-X(t)| \\
& \leq\left(K_{1}+K_{2}\right)|x-t|^{g(X(t))-c-\delta}+\frac{1}{4}|X(x)-X(t)|
\end{aligned}
$$

where we have used (40). Finally, from (48) and (49), one obtains that, for all $x, t \in$ $[0,1],|x-t|<\epsilon$ :

$|X(t)-X(x)| \leq\left|X_{N-1}(t)-X_{N-1}(x)\right|+2\left(K_{1}+K_{2}\right)|x-t|^{g(X(t))-c-\delta}+\frac{1}{2}|X(x)-X(t)|$,

or

$$
|X(t)-X(x)| \leq 2\left|X_{N-1}(t)-X_{N-1}(x)\right|+4\left(K_{1}+K_{2}\right)|x-t|^{g(X(t)-c-\delta} .
$$


When $x \rightarrow t,\left|X_{N-1}(t)-X_{N-1}(x)\right|=O(|t-x|)=o\left(|t-x|^{g(X(t))-c-\delta)}\right)$, and thus $|X(t)-X(x)|=O\left(|t-x|^{g(X(t)-c-\delta)}\right)$, which entails that $\alpha_{X}^{l}(t) \geq g(X(t))-c-\delta$ for all $\delta>0$, that is $\alpha_{X}^{l}(t) \geq g(X(t))-c$.

Let us now move to the proof of the upper bound on $\alpha_{X}(t)$ under Assumption (22). We will need the following lemma:

Lemma 6.5. Let $\left(Z_{j k}\right)_{k=0.2^{j}-1, j \in \mathbb{N}}$ be i.i.d centred random variables satisfying Assumption 22]. Let $\epsilon>0$. Then, almost surely, for all $x \in[0,1]$, there exists a sequence $\left(j_{n}, k_{n}\right)_{n}$ with $j_{n}$ tending to infinity, such that, for all $n, x \in$ $\left[k_{n} 2^{-j_{n}},\left(k_{n}+1\right) 2^{-j_{n}}\right)$ and $\left|Z_{j_{n} k_{n}}\right| \geq 2^{-j_{n} \epsilon}$.

Proof of Lemma 6.5.

Fix for now $j \in \mathbb{N}$ and $k \in\left[0 \cdots 2^{j}-1\right]$.

Let us give an upper bound to the probability that $\left|Z_{l m}\right|$ is smaller than $2^{-l}$ for a couple $(l, m)$ "below" $(j, k)$ (that is, such that $\left.m 2^{-l} \in\left[k 2^{-j} ;(k+1) 2^{-j}\right)\right)$. For any $l \geq j$, consider the event

$$
\Omega_{l}^{j, k}=\left\{\exists m: m 2^{-l} \in\left[k 2^{-j},(k+1) 2^{-j}\right) \text { and }\left|Z_{l m}\right| \leq 2^{-l \epsilon}\right\} .
$$

Since $P\left(\left|Z_{l m}\right| \leq 2^{-l \epsilon}\right) \leq 2^{-l \gamma \epsilon}$, one has $P\left(\Omega_{l}^{j, k}\right) \leq 2^{l-j} 2^{-l \gamma \epsilon}$. Applying this with $l+j$ in place of $l$, one gets, for $l \geq 0$,

$$
P\left(\Omega_{j+l}^{j, k}\right) \leq 2^{-j \gamma \epsilon} 2^{(1-\gamma \epsilon) l} .
$$

Fix $\beta \in(0,1)$, and denote: $W_{j, k}=\bigcap_{l=0}^{\left[j^{\beta}\right\rceil} \Omega_{j+l}^{j, k} . W_{j, k}$ is the event: "at each scale $l$ with $j \leq l \leq j+j^{\beta}$, there exists at least one coefficient $\left|Z_{l m}\right|$ such that $m 2^{-l} \in\left[k 2^{-j},(k+1) 2^{-j}\right]$ and $\left|Z_{l m}\right| \leq 2^{-l \epsilon "}$.

Using independence of the events $\left(\Omega_{j+l}^{j, k}\right)$, one has:

$$
\begin{aligned}
P\left(\bigcap_{l=0}^{\left\lceil j^{\beta}\right\rceil} \Omega_{j+l}^{j, k}\right) & =\prod_{l=0}^{\left\lceil j^{\beta}\right\rceil} P\left(\Omega_{j+l}^{j, k}\right) \\
& \leq 2^{-j^{\beta+1} \gamma \epsilon} 2^{(1-\gamma \epsilon) \sum_{l=0}^{\left\lceil j^{\beta}\right\rceil} l} \\
& \leq 2^{-j^{\beta+1} \gamma \epsilon} 2^{(1-\gamma \epsilon) j^{2 \beta}} .
\end{aligned}
$$

Letting now $j$ and $k$ vary, consider the event $V_{j}=\bigcup_{k=0}^{2^{j}-1} W_{j, k}$. Then,

$$
\begin{aligned}
P\left(V_{j}\right) & \leq \sum_{k=0}^{2^{j}-1} P\left(W_{j, k}\right) \\
& \leq 2^{j^{2 \beta}+j-j^{\beta+1} \gamma \epsilon} .
\end{aligned}
$$

Since $\beta \in(0,1)$, Borel-Cantelli Lemma then entails that, almost surely, the event $V_{j}$ occurs only for a finite set of indices $j$. In other words, almost surely, there exists $J$ such that, for all $j \geq J$ and all $k \in\left[0 \cdots 2^{j-1}\right]$, there exists $l \geq j$ such that all the random variables $Z_{l m}$ with $m 2^{-l} \in\left[k 2^{-j},(k+1) 2^{-j}\right]$ are larger in absolute value than $2^{-l \epsilon}$.

Consider now $x \in[0,1]$. Choose $j \geq J$ and $k$ such that $x \in\left[k 2^{-j},(k+1) 2^{-j}\right)$. From what we have just proved, we know that there exists $j_{1} \geq j$ such that for all 
$m$ with $m 2^{-j_{1}} \in\left[k 2^{-j},(k+1) 2^{-j}\right)$ one has: $\left|Z_{j_{1} m}\right| \geq 2^{-j_{1} \epsilon}$. Define $k_{1}$ to be the integer such that $x \in\left[k_{1} 2^{-j_{1}},\left(k_{1}+1\right) 2^{-j_{1}}\right)$. To obtain $j_{2}$ and $k_{2}$, one iterates the same process, replacing $j$ by $j_{1}+1$. In this way, one constructs a sequence $\left(j_{n}, k_{n}\right)_{n}$ which is easily seen to fulfil the statements of the Lemma.

Let us go back to the proof of Theorem 4.1.

Fix $\epsilon>0$. Using Lemma 6.5, almost surely, for all $x \in[0,1]$, there exists a sequence $\left(j_{n}, k_{n}\right)_{n}$ where $\left(j_{n}\right)_{n}$ tends to infinity and such that $x \in\left[k_{n} 2^{-j_{n}},\left(k_{n}+\right.\right.$ 1) $\left.2^{-j_{n}}\right)$ and $\left|Z_{j_{n} k_{n}}\right| \geq 2^{-j_{n} \epsilon}$.

Let: $m_{n}=\left(k_{n}+1 / 2\right) 2^{-j_{n}}$ and $M_{n}=\frac{X\left(k_{n} 2^{-j_{n}}\right)+X\left(\left(k_{n}+1\right) 2^{-j_{n}}\right)}{2}$.

By definition of $X$,

$$
X\left(m_{n}\right)-M_{n}=X_{j_{n}}\left(m_{n}\right)-M_{n}=Z_{j_{n} k_{n}} 2^{-j_{n} g\left(X_{j_{n-1}}\left(m_{n}\right)\right)},
$$

and, by definition of $M_{n}$, at least one of the two following inequalities hold:

$$
\begin{aligned}
\left|X\left(m_{n}\right)-X\left(\left(k_{n}+1\right) 2^{-j_{n}}\right)\right| & \geq\left|Z_{j_{n} k_{n}} 2^{-j_{n} g\left(X_{j_{n-1}}\left(m_{n}\right)\right)}\right|, \\
\text { or: } & \\
\left|X\left(m_{n}\right)-X\left(\left(k_{n}\right) 2^{-j_{n}}\right)\right| & \geq\left|Z_{j_{n} k_{n}} 2^{-j_{n} g\left(X_{j_{n-1}}\left(m_{n}\right)\right)}\right| .
\end{aligned}
$$

Using the property of the sequence $\left(j_{n}, k_{n}\right)_{n}$, one deduces:

$$
\begin{aligned}
\left|X\left(m_{n}\right)-X\left(\left(k_{n}+1\right) 2^{-j_{n}}\right)\right| & \geq 2^{-j_{n}\left(g\left(X_{j_{n-1}}\left(m_{n}\right)\right)+\epsilon\right)}, \\
\text { or: } & \\
\left|X\left(m_{n}\right)-X\left(\left(k_{n}\right) 2^{-j_{n}}\right)\right| & \geq 2^{-j_{n}\left(g\left(X_{j_{n-1}}\left(m_{n}\right)\right)+\epsilon\right)} .
\end{aligned}
$$

Since $m_{j_{n}, k_{n}}$ tends to $x$, we have, using the facts that $X_{j_{n}}$ tends uniformly to $X$ and that $g$ is continuous:

$$
\exists N \in \mathbb{N}: \forall n \geq N, g\left(X_{j_{n-1}}\left(m_{j_{n}, k_{n}}\right)\right) \leq g(x)+\epsilon .
$$

Thus, for $n \geq N$,

$$
\begin{aligned}
\left|X\left(m_{n}\right)-X\left(\left(k_{n}+1\right) 2^{-j_{n}}\right)\right| & \geq 2^{-j_{n}(g(X(x))+2 \epsilon)}, \\
\text { or: } & \\
\left|X\left(m_{n}\right)-X\left(\left(k_{n}\right) 2^{-j_{n}}\right)\right| & \geq 2^{-j_{n}(g(X(x))+2 \epsilon)} .
\end{aligned}
$$

Among the three numbers $X\left(m_{n}\right), X\left(k_{n} 2^{-j_{n}}\right)$ and $X\left(\left(k_{n}+1\right) 2^{-j_{n}}\right)$, two at least are within a distance not smaller than $2^{-j_{n}(g(X(x))+2 \epsilon)}$. As a consequence, one at least is at distance from $X(x)$ not smaller than $\frac{1}{2} 2^{-j_{n}(g(X(x))+2 \epsilon)}$. Let $x_{n}$ denote one of the abscissa such that $\left|X\left(x_{n}\right)-X(x)\right| \geq \frac{1}{2} 2^{-j_{n}(g(X(x))+2 \epsilon)}$ (thus $x_{n}$ is equal to either $m_{n}, k_{n} 2^{-j_{n}}$ or $\left.\left(k_{n}+1\right) 2^{-j_{n}}\right)$.

One has $\left|x_{n}-x\right| \leq 2^{-j_{n}}$. This implies first that $x_{n}$ tends to $x$ when $n$ tends to infinity. Second, for $n>N$ :

$$
\begin{aligned}
\left|X\left(x_{n}\right)-X(x)\right| & \geq \frac{1}{2} 2^{-j_{n}(g(X(x))+2 \epsilon)} \\
& \geq \frac{1}{2}\left|x_{n}-x\right|^{g(X(x))+2 \epsilon} .
\end{aligned}
$$

This entails that $\alpha_{X}(x) \leq g(X(x))+2 \epsilon$, an inequality which is true for all $\epsilon>0$ and thus $\alpha_{X}(x) \leq g(X(x))$.

This ends the proof of Theorem 4.1 . 


\section{References}

[1] A. Ayache and J. Lévy Véhel, The generalized multifractional brownian motion, Statistical Inference for Stochastic Processes, 3(1/2), 7-18, 2000.

[2] S. Bianchi and A. Pianese, Modelling stock price movements: multifractality or multifractionality?, Quantitative Finance, 7(3), 301-319, 2007.

[3] A. Benassi, S. Jaffard and D. Roux, Elliptic Gaussian random processes, Revista Mathemàtica Iberoamericana, 13(1), 19-90, 1997.

[4] P. Legrand and J. Lévy Véhel, Hölderian regularity-based image interpolation, ICASSP06, International Conference on Acoustics, Speech, and Signal Processing, 2006.

[5] K.J. Falconer, The local structure of random processes, Journal of the London Mathematical Society, 67, 657-672, 2003.

[6] K.J. Falconer, Localisable, multifractional and multistable processes, Séminaires et Congrès, 28, 1-12, 2012.

[7] A.N. Kolmogorov, Wienersche Spiralen und einige andere interessante Kurven in Hilbertchen Raume, Doklady, 26, 115-118, 1940.

[8] A. Ayache and M. Taqqu, Multifractional process with random exponent, Publicationes Mathematicae, 49, 459-486, 2005.

[9] A.Ayache, S. Cohen and J. Lévy Véhel, The covariance of the multifractional Brownian motion and applications to long-range dependence, ICASSP00, International Conference on Acoustics, Speech, and Signal Processing, 2000.

[10] B. Mandelbrot and J. Van Ness, Fractional Brownian motion, fractional noises and applications, SIAM Review, 10, 422-437, 1968.

[11] K. Daoudi, J. Lévy Véhel, and Y. Meyer, Construction of continuous functions with prescribed local regularity, Constructive Approximation, 014(03), 349385, 1996.

[12] R.F. Peltier and J. Lévy Véhel, Multifractional Brownian motion: definition and preliminary results, INRIA Research Report 2645, 1995. http://hal.inria.fr/docs/00/07/40/45/PDF/RR-2645.pdf

[13] S. Stoev and M.S. Taqqu, How rich is the class of multifractional Brownian motions?, Stochastic Processes and their Applications, 116, 200-211, 2006.

[14] C. Tricot, Curves and Fractal Dimension, Second Edition, Springer Verlag, 1995.

[15] FracLab, a MatLab toolbox for signal and image analysis. Available at http://fraclab.saclay.inria.fr/.

[16] A. Echelard, O. Barrière and J. Lévy Véhel, Terrain modelling with multifractional Brownian motion and self-regulating processes, Lecture Notes in Computer Science, 6374, 342-351, Springer, 2010.

[17] O. Barrière and J. Lévy Véhel, Intervalles interbattements cardiaques et Processus Auto-Régulé Multifractionnaire, Journal de la Société Française de Statistique, 150 (1), 54-72.

[18] A. Echelard and J. Lévy Véhel, Self-regulating processes-based modelling for arrhythmia characterization, ISPHT 2012, International Conference on Imaging and Signal Processing in Health Care and Technology, 2012. 
[19] A. Echelard, J. Lévy Véhel and A. Philippe, Estimating the self-regulating function of self-regulating midpoint displacement processes, preprint, 2012.

[20] R. Fischer, M. Akay, P. Castiglioni and M. Di Rienzo, Multi- and monofractral indices of short-term heart variability, Medical \& Biological Engineering \& Computing, 5 (41), 543-549, 2003.

[21] K. Weierstrass, On Continuous Function of a Real Argument that do not have a Well-Defined Differential Quotient, Mathematische Werke, 2, 71-74, 1895.

[22] G.H. Hardy, On Weierstrass's NonDifferentiable Function, Transaction of the American Mathematical Society, 17, 301-325, 1916.

[23] A. Wood and G. Chan, Simulation of stationary Gaussian processes in $[0,1]^{d}$, Journal of Computational and Graphical Statistics, 3 (4), 409-432, 1994. 